\title{
Modular Network between Postrhinal Visual Cortex, Amygdala, and Entorhinal Cortex
}

\author{
${ }^{(1)}$ Andrew M. Meier, Quanxin Wang, Weiqing Ji, Jehan Ganachaud, and Andreas Burkhalter \\ Department of Neuroscience, Washington University School of Medicine in St. Louis, St. Louis, Missouri 63110
}

The postrhinal area (POR) is a known center for integrating spatial with nonspatial visual information and a possible hub for influencing landmark navigation by affective input from the amygdala. This may involve specific circuits within muscarinic acetylcholine receptor $2(\mathrm{M} 2)$-positive $\left(\mathrm{M}^{+}\right)$or $\mathrm{M}^{-}$modules of POR that associate inputs from the thalamus, cortex, and amygdala, and send outputs to the entorhinal cortex. Using anterograde and retrograde labeling with conventional and viral tracers in male and female mice, we found that all higher visual areas of the ventral cortical stream project to the amygdala, while such inputs are absent from primary visual cortex and dorsal stream areas. Unexpectedly for the presumed saltand-pepper organization of mouse extrastriate cortex, tracing results show that inputs from the dorsal lateral geniculate nucleus and lateral posterior nucleus were spatially clustered in layer 1 (L1) and overlapped with $\mathrm{M}^{+}$patches of POR. In contrast, input from the amygdala to L1 of POR terminated in M2 ${ }^{-}$interpatches. Importantly, the amygdalocortical input to $\mathrm{M}^{-}$interpatches in L1 overlapped preferentially with spatially clustered apical dendrites of POR neurons projecting to amygdala and entorhinal area lateral, medial (ENTm). The results suggest that subnetworks in POR, used to build spatial maps for navigation, do not receive direct thalamocortical $\mathrm{M}^{+}$patch-targeting inputs. Instead, they involve local networks of $\mathrm{M}^{-}$ interpatches, which are influenced by affective information from the amygdala and project to ENTm, whose cells respond to visual landmark cues for navigation.

Key words: amygdala; entorhinal cortex; navigation; postrhinal cortex; thalamocortical connections; visual cortex

Significance Statement

A central purpose of visual object recognition is identifying the salience of objects and approaching or avoiding them. However, it is not currently known how the visual cortex integrates the multiple streams of information, including affective and navigational cues, which are required to accomplish this task. We find that in a higher visual area, the postrhinal cortex, the cortical sheet is divided into interdigitating modules receiving distinct inputs from visual and emotion-related sources. One of these modules is preferentially connected with the amygdala and provides outputs to entorhinal cortex, constituting a processing stream that may assign emotional salience to objects and landmarks for the guidance of goal-directed navigation.

\section{Introduction}

The chief concern for survival is to detect danger and react to it (Price, 2005). In rodents, defensive strategies include the reflexive startle response, freezing when danger is distant and escaping when it is close. To do this, it is important to know the spatial layout of the environment. For this, animals rely on neural

\footnotetext{
Received Aug. 10, 2020; revised Apr. 2, 2021; accepted Apr. 7, 2021.

Author contributions: A.M.M., Q.W., and A.B. designed research; A.M.M., Q.W., W.J., and A.B. performed research; A.M.M., J.G., and A.B. analyzed data; A.M.M. and A.B. wrote the paper.

This research was supported by National Eye Institute Grants R01-EY-016184, R01-EY-020523, R01-EY027383, and R21-EY-027946; and the McDonnell Center for Systems Neuroscience. We thank Katia Valkova for technical support.

A. Meier's present address: Boston University College of Engineering, Boston, MA 02215.

Q. Wang's present address: Allen Institute for Brain Science, Seattle, WA 98109.

The authors declare no competing financial interests.

Correspondence should be addressed to Andreas Burkhalter at burkhala@wustl.edu.

https://doi.org/10.1523/JNEUROSCI.2185-20.2021

Copyright $\odot 2021$ the authors
}

circuits connecting multiple brain structures including the amygdala, which drives flight, freezing, and approach (Janak and Tye, 2015); and areas of the limbic and sensory cortex (LeDoux, 2012). In rodents, a critical structure is the postrhinal area (POR; Burwell, 2001), which is part of the parahippocampal cortex (Burwell et al., 1995) and is a center for visual scene processing (Epstein et al., 1999). Studies in rats have shown that POR integrates visual inputs to extract contextual information (Furtak et al., 2012). It also references head-centered information to the visual scene, which is thought to form the basis for the grid-cell metric in entorhinal cortex (Furtak et al., 2012; Gofman et al., 2019; LaChance et al., 2019). The network for spatial processing is often contrasted with a pathway that carries nonspatial information through perirhinal and lateral entorhinal cortices to the hippocampus (Deshmukh et al., 2012; Knierim et al., 2014; Nilssen et al., 2019). The second function is the association of visual objects with reward and fear memory via a circuit with the amygdala (Agster et al., 2016; Burgess et al., 2016; Ramesh et al., 
2018). This pathway is known as the "cortical route" for visual fear response, as opposed to the "subcortical route" for aversive responses (Pessoa and Adolphs, 2011; Agster et al., 2016). A source of the subcortical input channel is the superior colliculus (SC), which connects through the lateral posterior nucleus to the amygdala, where it triggers defensive responses to visual threats (Wei et al., 2015). This information may be used in the circuit with POR to associate affective significance to objects and places (Furtak et al., 2012; Burgess et al., 2016; Ramesh et al., 2018). A second branch of the pulvinocortical pathway sends input directly to POR and provides for its high sensitivity to moving stimuli (Nishio et al., 2018; Beltramo and Scanziani, 2019). Calcium recordings in $\mathrm{V} 1$ have shown that lateral posterior nucleus (LP) terminals carry signals for unexpected visual motion that are incongruent with the running speed of the animal (Roth et al., 2016). These responses suggest that locomotion-related cues may be integrated in POR, enabling the detection of moving objects during self-motion. Direct input to POR may also come from the dorsal lateral geniculate nucleus (dLGN; experiment 479670988, Allen Brain Atlas), whose terminals in layer 1 (L1) of primary visual cortex (V1) are most active when the running speed matches the optic flow (Roth et al., 2016), providing cues for determining the position relative to landmarks.

We have shown in mouse POR that muscarinic acetylcholine receptor 2 (M2) expression in L1 is patchy (Ji et al., 2015). Recordings in V1 have further shown that L2/3 neurons aligned with $\mathrm{M} 2^{+}$patches are more sensitive to object features, while cells in $\mathrm{M}^{-}$interpatches are more sharply tuned to moving stimuli (Ji et al., 2015). This pattern is reflected in the organization of dLGN inputs to $\mathrm{M}^{+}$patches and LP projections to $\mathrm{M}^{-}$interpatches (D'Souza et al., 2019). The findings raised the question whether the modular organization of thalamocortical inputs is preserved in POR, and inputs from the amygdala overlap with neurons projecting to the amygdala and ENTm. To address these questions, we have used anterograde and retrograde tracers of connections to and from POR. The results show that POR receives inputs mainly from areas of the ventral cortical processing stream (Wang et al., 2012). dLGN and LP projections to L1 overlap in $\mathrm{M}^{+}$patches, whereas inputs from the amygdala terminate in $\mathrm{M}^{-}$interpatches. The overlap of amygdalocortical inputs with the apical dendritic tufts of POR neurons projecting to the amygdala and ENTm suggests that $\mathrm{M} 2^{-}$modules provide a substrate for the affective modulation of POR output used for navigation.

\section{Materials and Methods}

\section{Animals}

Experiments were performed in 5- to 10-week-old male and female C57BL/ 6), Ai9 (Gt[ROSA]26Sor ${ }^{\text {tm9(CAG-tdTTomato)Hze }) / J, ~ P V-C r e ~(B g .129 P 2-~}$ Pvalb $\left.^{\text {Tm(CRE)Arbr }}\right) / \mathrm{J}$, x Ai9 (Gt[ROSA 26 Sor $^{\text {tm9(CAG-tdTTomato)Hze }) / J \text {, and }}$ ChrM2 ${ }^{-}$tdT-D knock-in (BG6.Cg-Chrm2 $\left.2^{\text {tm1.1Hze }}\right) /$ J mice. All experimental procedures were performed in accordance with the National Institutes of Health guidelines and under the approval of the Washington University Institutional Animal Care and Use Committee.

\section{Surgical procedures}

Mice were anesthetized with a ketamine/xylazine mixture $(86 / 13 \mathrm{mg} / \mathrm{kg}$, i.p.). Analgesia was achieved by presurgical injections of buprenorphineSR $(0.1 \mathrm{mg} / \mathrm{kg}$, s.c.). Ocular ointment was applied to protect the corneas. Mice were head fixed in a stereotaxic frame. Body temperature was maintained at $37^{\circ} \mathrm{C}$. For each injection site, a small craniotomy was made over the target. Tracer injections were performed with glass micropipets (tip diameter, 15-20 $\mu \mathrm{m}$ ) and, depending on the tracer used, attached to a Picospritzer [for bisbenzimide and diamidino yellow
(DY)], an iontophoresis current source [for biotinylated dextran amine (BDA)], or a Nanoject II pump [for adeno-associated viruses (AAVs)]. At each cortical site, two injections were made: one at $0.3 \mathrm{~mm}$ and the other at $0.5 \mathrm{~mm}$ below the pial surface. After injections were complete, pipettes remained in place for $3 \mathrm{~min}$ before they were retracted. The scalp was stapled closed with wound clips.

\section{Combined anterograde tracing with BDA and retrograde tracing with} bisbenzimide

For mapping axonal projections from visual cortex to the amygdala in C57BL/6J mice, BDA (10,000 molecular weight, $5 \%$ in $\mathrm{H}_{2} \mathrm{O}$; Thermo Fisher Scientific) was injected through a glass micropipet using iontophoresis (7 s duty cycle for $7 \mathrm{~min}, 3 \mu \mathrm{A}$; Midgard, Stoelting). Injections were made into the left hemisphere (in $\mathrm{mm}$, anterior to transverse sinus/ lateral to midsagittal suture), as follows: V1 (1.1/2.8), lateromedial area $(\mathrm{LM} ; 1.4 / 4.0)$, posterior area $(\mathrm{P} ; 1.0 / 4.2)$, laterointermediate area (LI; $1.45 / 4.2)$, POR (1.15/4.3), anterolateral area (AL; $2.4 / 3.7)$, rostrolateral area $(\mathrm{RL} ; 2.8 / 3.3)$, posteromedial area $(\mathrm{PM} ; 1.9 / 1.6)$, anteromedial area (AM; 3.0/1.7), and anterior area (A; 3.4/2.4). In the same animal, callosal connections were retrogradely labeled by blanketing the right visual cortex with 20-30 injections (20 nl each) of bisbenzimide $\left(5 \%\right.$ in $\mathrm{H}_{2} \mathrm{O}$; Sigma-Aldrich). After $3 \mathrm{~d}$ of survival, mice were killed with an overdose of ketamine/xylazine $(500 / 50 \mathrm{mg} / \mathrm{kg}$, i.p.) and perfused with $4 \%$ paraformaldehyde [PFA; $0.1 \mathrm{~m}$ phosphate buffer (PB), $\mathrm{pH}$ 7.4]. The brain was extracted from the cranium and cryoprotected in $30 \%$ sucrose (PB). The bisbenzimide-labeled callosal projection pattern was imaged in situ under a stereomicroscope (model MZ16F, Leica) equipped with fluorescence optics (excitation, $338 \mathrm{~nm}$; emission, $505 \mathrm{~nm}$ ). Injection sites were visible in situ (and validated later in sections) as weakly fluorescent spots (see Fig. $3 A-J$ ), whose locations relative to the callosal labeling pattern were used for areal identification (Wang and Burkhalter, 2007). Coronal sections $(50 \mu \mathrm{m})$ were cut on a cryostat and stained for BDA using an avidin-biotin complex (ABC) reaction (Vectastain ABC Elite System). Sections containing the brainstem, diencephalon, and the amygdala were mounted on glass slides, and after dehydration in ethanol and clearing in xylenes, the diaminobenzidine reaction product was intensified with $\mathrm{AgNO}_{3}$ and $\mathrm{HAuCl}$ (Wang et al., 2012). Representative sections were stained with cresyl violet for Nissl substance. The slides were coverslipped with mounting medium (DPX, Sigma-Aldrich) and imaged under a microscope equipped with dark-field optics. Images were captured with a CCD camera (CoolSnap EZ, Roper Scientific). For analyses of the termination pattern in each nucleus, coronal sections were imaged under $10 \times$ magnification, and grayscale images were recorded with MetaMorph NX2.0 acquisition software (Molecular Devices). The optical density (OD) of BDA-labeled axons was determined by blurring the raw image and measuring pixel values in regions of interest (ROIs) with ImageJ.

\section{Retrograde tracing with diamidino yellow}

To determine the sources of cortical and subcortical inputs to POR, neurons were retrogradely labeled with the fluorescent tracer diamidino yellow (2\% DY in $\mathrm{H}_{2} \mathrm{O}, 50 \mathrm{nl}$; EMS-Chemie). Injections into POR (1.15/4.3 $\mathrm{mm}$ anterior to transverse sinus/lateral to midsagittal suture) were made in PVtdT (parvalbumin-tdT) mice whose pattern of tdT (tdTomato) fluorescence was used to identify cortical areas and subcortical nuclei (Gămănuţ et al., 2018). After $3 \mathrm{~d}$ of survival, mice were perfused with $1 \%$ PFA. Brains were extracted, and the cortex including the hippocampus were detached from the rest of the brain and flatmounted (Ji et al., 2015). Next, the tissue was postfixed in $4 \%$ PFA (overnight, at $4^{\circ} \mathrm{C}$ ), followed by overnight immersion in $30 \%$ sucrose (in $\mathrm{PB}$, at $4^{\circ} \mathrm{C}$ ). Tangential sections were cut at $40 \mu \mathrm{m}$ on a freezing microtome, wet mounted on glass slides, and coverslipped in PB. Images were captured with a CCD camera (Lumenera InfinityS3-URM, Teledyne) and MetaMorph NX2.0 acquisition software (Molecular Devices) using a stereomicroscope (model MZ16F, Leica) or an epifluorescence microscope (model 80i, Nikon), respectively, equipped with UV fluorescence (excitation, 355-425 nm; emission, $470 \mathrm{~nm}$ ) and tdT (excitation, 520$600 \mathrm{~nm}$; emission, $570-720 \mathrm{mn}$ ) optics. For storage, sections were mounted on glass slides and coverslipped with DPX. To reveal DY- 
labeled cells in the amygdala, the neocortex was separated along the rhinal fissure from the rest of the brain, which was then sectioned at $40 \mu \mathrm{m}$ in the coronal plane.

\section{Anterograde tracing with $A A V s$}

For retinotopic mapping of POR in C57BL/6J mice, side-by-side injections with AAV.2/1.hSynapsin.EGFP.WPRE.bGH (46 nl; Penn Vector Core) and AAV.2/1.hSynapsin.tdTomato.WPRE (46 nl; Penn Vector Core) were made at select combinations of retinotopic locations in V1. The injections were confined to a space within (in $\mathrm{mm}$, anterior to transverse sinus/lateral to midsagittal suture) $1.0-1.6 / 2.7-1.4 / 3.3$. The same viral tracers and injection volumes were used to trace inputs from the subcortical structures (in $\mathrm{mm}$, posterior of bregma/lateral of midline/ below pial surface) lateral amygdala (LA; $1.8 / 3.55 / 3.7)$, dLGN (2.35/2.15/ $2.55)$, and LP (1.85/1.25/2.65) in C57BL/6J or Chrm2-tdT mice. After $14-18 \mathrm{~d}$ of survival mice were killed and perfused with $1 \%$ PFA. The cortex of V1-, dLGN-, and LP-injected mice was flatmounted, postfixed in $4 \%$ PFA, cryoprotected in $30 \%$ sucrose, and stored overnight with the rest of the brain. In amygdala-injected mice, neocortex was separated from the limbic region along the rhinal fissure, flatmounted, postfixed, cryoprotected, and sectioned at $40 \mu \mathrm{m}$ in the tangential plane on a freezing microtome. The amygdala was sliced in the coronal plane. For visualization of M2 expression in C57BL/6J mice, tangential, and coronal sections through cortex were immunostained with an antibody against M2. This procedure involved preincubation in blocking solution $(0.1 \%$ Triton X-100, 10\% normal goat serum, PB), followed by incubation in primary rat anti-M2 antibody (1:500, for $48 \mathrm{~h}$ at $4^{\circ} \mathrm{C}$; catalog \#MAB367, Millipore) and reaction with Alexa Fluor 647-labeled goat anti-rat secondary antibody (1:500; catalog \#A21247, Thermo Fisher Scientific). M2 labeling patterns in Chrm2-tdT mice were identified by tdT fluorescence. Selected sections through cortex and amygdala were counterstained with Invitrogen NeuroTrace 435/455 (Thermo Fisher Scientific). Anterogradely labeled axons and M2 patches were imaged at 4-40× under an epifluorescence microscope equipped with extended green fluorescent protein (EGFP), tdT, and infrared (IR; excitation, $650 \mathrm{~nm}$; emission, $665 \mathrm{~nm}$ ) fluorescence optics, a CCD camera, and acquisition software. In cortical sections, POR and PORa (anterior POR) were outlined based on M2 expression. Above-background EGFP or tdT fluorescence intensity in POR, LM, and LI was determined in series of 2-12 tangential sections.

To disambiguate laminar identification based on cytoarchitecture (i.e., counterstaining with NeuroTrace 435/455) in flatmounted cortex, we immunostained tangential sections of flatmounted cortex of C57BL/6J mice with an antibody against the transcription factor CTIP2 (COUP TFinteraction protein), which selectively labels L5 and L6 (Arlotta et al., 2005). Immunostaining was performed with a rat anti-CTIP2 antibody (1:500; catalog \#18465, Abcam), which was visualized with an Alexa Fluor 488-labeled secondary goat anti-rat IgG (1:500, Thermo Fisher Scientific).

\section{Retrograde tracing with AAVs}

To investigate the tangential distribution of POR neurons that project to the amygdala and the entorhinal cortex, we used retrograde tracing with rAAV2-Retro/CAG-Cre (University of North Carolina Vector Core) in $\mathrm{Ai} 9$ mice. Injections ( $46 \mathrm{nl}$ ) were made into the $\mathrm{LA}$ (in $\mathrm{mm}$, posterior of bregma/lateral of midline/below pial surface, 1.8/3.55/3.7) and the ENTm (in mm, anterior to transverse sinus/lateral to midline/below the pial surface, $0.7 / 5.25 / 3.55$ ) angled $40^{\circ}$ from vertical. After $14-18 \mathrm{~d}$ of survival, perfusion with $1 \%$ PFA, flatmounting of cortex, postfixation in $4 \%$ PFA, cryoprotection, and cutting in the tangential plane, sections were immunostained with rat anti-M2 and Alexa Fluor 647-labeled goat antirat antibodies. Sections were wet mounted on glass slides and imaged under IR and tdT illumination at 4-40× magnification to identify M2 patches, cell bodies, dendrites, spines, and local axons (Vaughan and Peters, 1973; Karimi et al., 2020) of retrogradely labeled cells in POR.

Patchy M2 expression

$\mathrm{M}^{+}$patches and $\mathrm{M} 2^{-}$interpatches were delineated from images of immunolabeling in C57BL/6J mice or the expression of Chrm2-tdT using custom MATLAB scripts. M2 expression images were spatially normalized by dividing the intensity of every pixel by the mean intensity within a $100 \mu \mathrm{m}$ radius. Images were blurred with the MATLAB "nanconv" function, a circular (pillbox) filter with $29 \mu \mathrm{m}$ radius (see Fig. $6 D$ ), and then divided into six quantiles based on pixel intensity (see Fig. 6E). These borders were overlaid onto images of virally anterogradely labeled axons or retrogradely labeled cell bodies and dendrites. Comparison of M2 immunostaining and Chmr2-tdT expression yielded nearly identical borders of $\mathrm{M}^{+}$patches and areal boundaries; the same analyses were therefore used for both labels. This similarity was verified by performing M2 immunostaining in Chrm2-tdT mice and overlaying M2 expression patterns from both markers in the same section.

\section{Experimental design and analyses}

Data were obtained from 44 C57BL/6J, 7 Chrm2tdT, 2 PVtdT, and 8 Ai9 adult male $(N=36)$ and female $(N=25)$ mice.

Optical density of BDA labeling. We determined the weight of anterogradely BDA-labeled projections by using ImageJ to measure the pixel values and compute the mean background-subtracted OD of axons (three sections/target/mouse). The OD in a specific nucleus was normalized to the sum of ODs measured in all of the 31 identified subcortical projection targets and expressed as averages across injection sites and mice. The relative strength of inputs from different visual areas to subnuclei of the amygdala was determined by computing the OD in the target relative to the maximal $\mathrm{OD}$ of the injection site. The data were plotted as the mean percentage OD of projections. The $t$ test was used for statistical comparisons.

Distribution and shape and of $\mathrm{M}^{+}$patches. For analysis of M2 patch distribution and shape, patch borders were drawn around the highest $20 \%$ intensity pixels and lowest $80 \%$ intensity pixels in blurred, highpass-filtered M2 expression images of tangentially sectioned L1. A highintensity threshold for pixel inclusion was used to ensure the separation of individual patches and to prevent the formation of large continuous units. To find out whether patches were anisotropic and aligned with the asymmetry of visual areas (see Fig. 2C,D), we first drew the outline of each area containing $\mathrm{M}^{+}$patches (V1, LM, LI, POR) based on M2 expression patterns in L4. These outlines were then used to determine the major and minor axes of visual areas, using custom scripts and the MATLAB "regiongroup" function. Patch anisotropy was quantified by the major/minor axis ratio. The spatial density of patches was calculated as units per square millimeter. Patches per square degree of visual space were determined by multiplying spatial densities with magnification factor of respective area (Garrett et al., 2014, their Fig. 7). The Tukey's test was used for statistical comparisons of patches in different areas.

Projection strength. For the quantification of the distribution of anterogradely labeled axonal projections in $\mathrm{M}_{2}^{+}$patches and $\mathrm{M}^{-}$interpatches, M2 expression images of tangential sections through L1 were high pass filtered, blurred, and divided into 6 intensity quantiles of equal area (see Fig. $6 D-F$ ). The lower three quantiles were treated as $\mathrm{M}^{-}$ interpatches and the upper three quantiles treated as $\mathrm{M}^{+}$patches. The strengths of anterogradely labeled LA/basolateral amygdala (BLA) $\rightarrow$ $\mathrm{POR}(N=5), \mathrm{dLGN} \rightarrow \mathrm{POR}(N=5)$, and $\mathrm{LP} \rightarrow \mathrm{POR}(N=2)$ projections in $\mathrm{M}^{+}$patches and $\mathrm{M} 2^{-}$interpatches of C57BL/6J and Chrm2tdT mice were determined using a custom-written MATLAB script to measure the pixel values of fluorescence intensity in ROIs $\left(360-630 \times 10^{3} \mu^{2}\right)$ and subtract the unlabeled background. For each projection target (POR, LM, LI, V1), the background-subtracted mean pixel values in $\mathrm{M}^{+}$patches and $\mathrm{M}^{-}$interpatches within the ROI were computed from one to two sections through L1. The laminar distribution of inputs to POR was compiled from sections through L2 to L6 in which registration to the patch/interpatch pattern of L1 was not available. Mean intensities in patches and interpatches and patch/interpatch intensity ratios were compared across quantiles using Pearson's correlation and one-sample $t$ test, respectively.

Distribution of dendrites. Images of dendrites labeled by retrograde tracing with rAAV2-Retro/CAG-Cre in Ai9 mice and M2-immunolabeled patches in L1 were acquired with a Leica Thunder Tissue Imager (model DMB 6) equipped with a camera (model DFC 9000 GT, Leica) and divided into six quantiles based on M2 expression intensity (see above). Apical dendrites in L1/2 of retrogradely labeled pyramidal cells 
in POR were distinguished from axons by greater thickness and were positively identified by their spiny morphology (Vaughan and Peters, 1973; Karimi et al., 2020). The dendrites were reconstructed at $40 \times$ magnification using Neurolucida (MBF Bioscience), and their total length within each quantile of M2 expression was computed. For each quantile in each mouse, the proportion of dendrite length of all dendrites within that image was determined, and the Pearson correlation coefficient between $\mathrm{M}^{-}$quantile level and the proportion of dendrites was computed. Images from four mice were analyzed for POR $\rightarrow$ ENTm projections and $\mathrm{POR} \rightarrow$ LA projections.

Laminar distribution of retrogradely labeled cells. Series of flatmounted tangential sections were taken from Ai9 mice in which rAAV2-Retro/CAG-Cre had been injected into either ENTm $(N=4$ mice) or LA ( $N=2$ mice). Images of M2 expression and retrogradely tdT-labeled cells across layers were taken at $4 \times$ and $10 \times$ magnification and overlaid. In each image, borders for LM, LI, P, POR, and PORa were manually drawn based on M2 immunolabeling visualized with Alexa Fluor 647 IgG, and all tdT-labeled cells were marked. Custom MATLAB scripts were used to tabulate counts of cells within each area. Cell counts for unusable sections were interpolated using Akima piecewise cubic interpolation with the MATLAB "interp1" function. To compensate for variations in labeling effectiveness across cases, cell counts were normalized for each mouse by dividing by the maximum number of cells in any one area in any one section of that mouse. For each mouse, the ratio of total cells labeled within a group within deep layers (L5 and L6 pooled together) to total cells labeled within L2/3 was computed.

Tangential clustering of retrogradely labeled cells. To quantify the clustering of cells in $\mathrm{M}^{+}$patches and $\mathrm{M}^{-}$interpatches of POR, retrogradely labeled from LA $(N=2)$ and ENTm $(N=4)$, we analyzed in tangential sections through L2/3 aligned with M2-immunostained patches in L1. Images were taken at $10 \times$ magnification (ROI, $160 \times 10^{3} \mu \mathrm{m}^{2}$ ) and divided into six quantiles based on M2 expression intensity. Cells were plotted manually and custom MATLAB scripts were used to count the number of cells in each M2 quantile. Cell counts were normalized within each image by dividing the cell count per quantile by the total number of cells labeled in that image. The Pearson correlation coefficient was then computed between quantile levels and normalized across multiple mice. Only images that contained $>10$ cells were analyzed.

\section{Data availability}

Code use for analysis in this study is available at https:/github.com/ amsmeier/Meier-et-al. 2021.

\section{Results \\ POR is contained within a $\mathrm{M2}^{+}$region of parahippocampal cortex}

The borders of POR have been outlined previously by mapping of retinotopic inputs from V1 (Wang and Burkhalter, 2007). However, it remained unknown whether its borders align with the expression of M2, an effective marker used to identify cortical areas (Gămănuţ et al., 2018). Additionally, the original description of POR in the studies by Burwell (2001) and Beaudin et al. (2013) was derived from cytoarchitectonic features observed in coronal sections. This raised the question whether the borders seen in coronal sections and flatmounted cortex are aligned with one another. To find out, we combined multicolor viral tracing of retinotopic projections from V1 with the expression patterns of M2 and PVtdT. We then analyzed the axonal projections in tangential sections through the flatmounted cortical hemisphere. Reconstructions of the retinotopic map were done from serial tangential sections through the cortical mantle, eliminating the challenging task of aligning sequential coronal sections. In tangential sections through L4 stained with an antibody against M2, we identified V1 as an intensely immunopositive triangular area surrounded by the more weakly fluorescent extrastriate cortex (Fig. 1A, inset). Lateral to V1, M2 stained a boot-shaped region that overlapped with the ventral stream areas LM (lateromedial), LI (laterointermediate), POR and PORa (Gămănuţ et al., 2018). M2 expression dropped sharply at a M2 $2^{-}$strip along the rhinal fissure, which contained ectorhinal area (ECT) and the posterior area $(\mathrm{P})$, and was continuous with the posterior temporal area (TEp), on the anterior-dorsal side of POR and PORa. A similar boot-shaped pattern was seen in the expression of PVtdT in PVcre x Ai9 mice (Fig. 1D), which overlapped with M2 (data not shown; Gămănuţ et al., 2018).

To outline the borders of areas within the $\mathrm{M}^{+}$boot, we simultaneously injected C57BL/6 J mice $(N=5)$ with two anterograde viral tracers, AAV.2/1.hSynapsin.EGFP (AAV.EGFP) and AAV.2/1.hSynapsin.tdTomato (AAV.tdT), at different visuotopic locations of V1. The result of one such case in which AAV. EGFP was injected near the intersection of the vertical and horizontal meridian (Garrett et al., 2014) and AAV.tdT was targeted to the upper temporal visual field (Wang and Burkhalter, 2007) is illustrated in Figure $1 A-C$. It shows that axonal projections terminated in multiple pairs of red and green patches distributed across the surrounding extrastriate cortex (Fig. 1B,C). Anteriordorsal to the rhinal fissure, we found three pairs of patches: two within the $\mathrm{M}^{+}{ }^{+}$boot and one in the $\mathrm{M} 2^{-}$strip. Reading the map from posterior to anterior starting at the border of V1, the colors of the projections switch from red to green, reverse back to red, and then reverse again to label a fainter patch of more interspersed red and green fibers. Because map reversals have been interpreted as areal borders (Sereno and Allman, 1991; Garrett et al., 2014), we concluded that the red-to-green/green-to-red and the green-to-red/red-to-green transitions mark the P/POR and the POR/PORa areal borders, respectively (Wang and Burkhalter, 2007; Wang et al., 2011). The systematic topographic shifts observed in five different mice suggest that $\mathrm{P}, \mathrm{POR}$, and PORa contain nearly complete maps of the visual field. The maps observed in two representative cases are depicted graphically in the inset of Figure $1 B$, in which the blue arrows indicate the progression from lower/nasal to upper/peripheral fields extracted from five different experiments. Together with previous findings that $\mathrm{P}, \mathrm{POR}$, and PORa have different connectivity profiles (Gămănuţ et al., 2018), these data suggest that these are distinct visual areas. We next asked whether each of these areas was contained within $\mathrm{M}^{+}$parahippocampal cortex or whether they extend across the border with ECT. We found that V1 injections at higher retinotopic altitudes shifted the anterogradely labeled patches in both POR and PORa closer to the borders with LM and LI, respectively (Fig. $1 B$, inset). In contrast, injections below the horizontal meridian pushed the projections backward, but never out of the $\mathrm{M}^{+}$region into the $\mathrm{M}^{-}$strip. This topographic pattern shows that the POR/ECT and PORa/ECT borders coincide with the posterior margin of the $\mathrm{M}^{+}$region, and indicates that PORa belongs to $\mathrm{M}^{+}$cortex and is not part of the agranular perirhinal area $36 \mathrm{p}$, as suggested previously (Wang and Burkhalter, 2007).

According to this scheme, postrhinal cortex is completely contained within the $\mathrm{M}^{+}$boot. This raised the question of whether the agranular ventral POR (PORv), identified in coronal sections by Beaudin et al. (2013), is part of postrhinal cortex. To find out, we looked at the cytoarchitecture of postrhinal cortex in Chrm2-tdT mice $(N=2)$. Figure $1, E$ and $F$, shows a coronal section from the approximate location indicated by the green line on the flatmap in Figure $1 \mathrm{~A}$. It shows that the multilayered M2 expression in the dorsal part is interrupted suddenly by an unstained gap and then reappears as a simpler pattern in more ventral parts of the section. In tangential sections, the unstained gap coincides precisely with the $\mathrm{M}^{-}$band aligned with the 

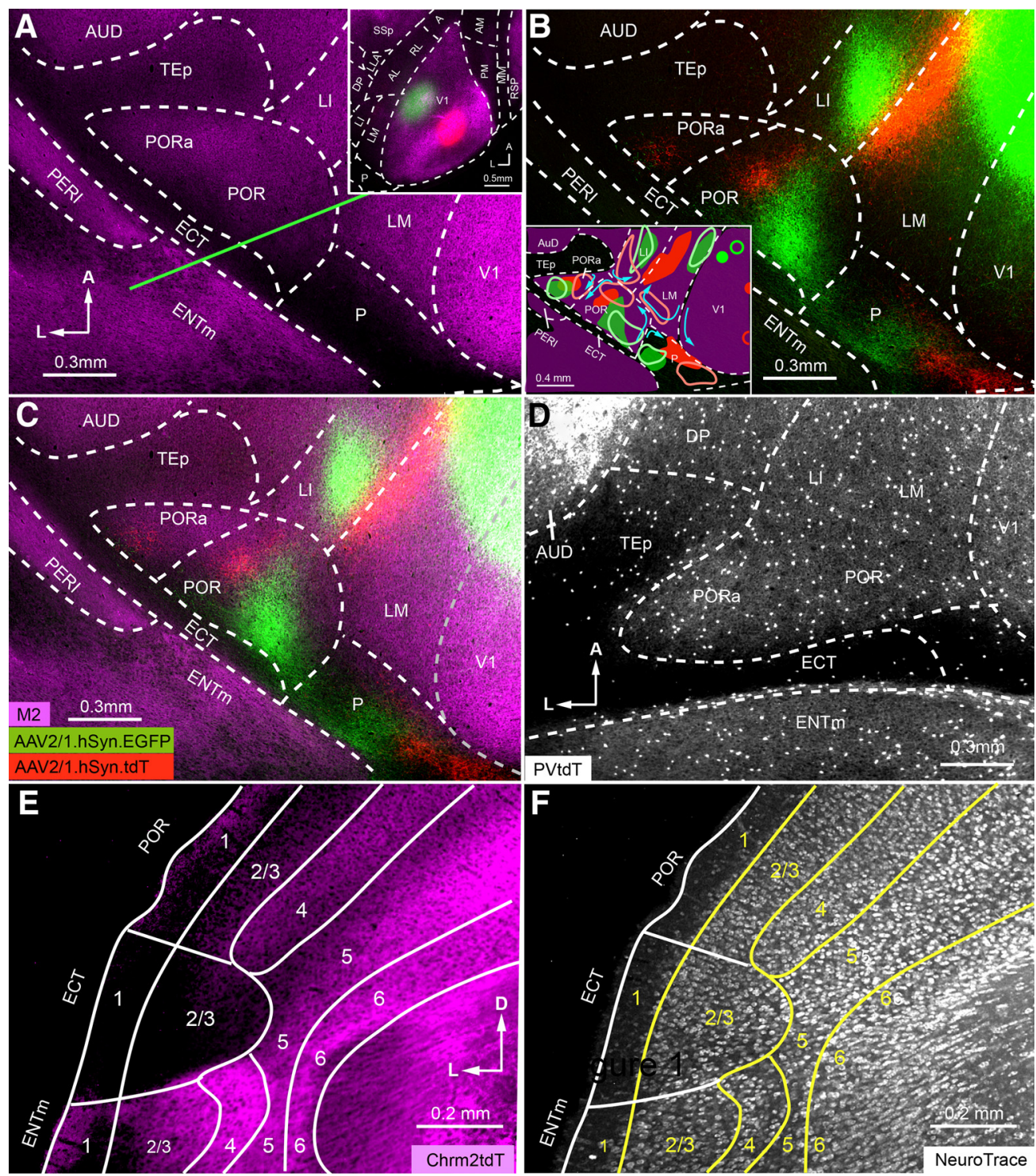

Figure 1. Mapping areas of mouse parahippocampal cortex. $A$, Tangential section through middle layers of flatmounted postrhinal cortex stained with an antibody against M2 muscarinic acetylcholine receptor (magenta). Inset shows low-magnification image of M2 expression in L4 of V1 and surrounding cortex. Green and red spots indicate AAV2/1.hSyn.EGFP (green) and AAV2/1.hSyn.tdT (red) injection sites into nasal/central and peripheral/upper visual field representations, respectively. $\boldsymbol{B}, \boldsymbol{C}$, Anterogradely labeled clusters of axon terminals labeled after AAV injections into V1 ( $\boldsymbol{A}$, inset) show retinotopic maps in LM, LI, P, POR, and PORa. Note that to show the projections in higher visual areas, the image was overexposed, which made the injection sites artificially large and gives the false impression that viral tracers spilled across the lateral V1/LM border. $\boldsymbol{B}$, inset, A diagram of visuotopic maps obtained from two representative experiments, whose V1 injections sites and projections are marked by solid and open green and red contours. Blue arrows denote the progression from lower/nasal to upper/peripheral visual field representations in different areas, derived from five different experiments. The overlay reveals that projection maps in POR and PORa are contained within the $\mathrm{ML}^{+}$cortex (C). $\boldsymbol{D}$, Expression of PVtdT in postrhinal cortex shows a foot-shaped pattern similar to M2 expression in $\boldsymbol{C} . M 2$ and PVtdT expression is extremely sparse in P, ECT, and TEp. $\boldsymbol{E}, \boldsymbol{F}$, Coronal section from Chmr2-tdT mouse (approximate location indicated by the green line in $\boldsymbol{A}$ ) shows sharp transitions of laminar M2 pattern at the POR/ECT and ECT/ENTm borders (E); after counterstaining with NeuroTrace 425/455, the transitions coincide with a loss of the granular appearance of $L 4$ and the distinctions with the larger cells in the more sparsely populated $L 5$ of $E C T$ were no longer apparent $(\boldsymbol{F})$. Axes: $\boldsymbol{A}-\mathbf{D}: A$, anterior; $L$, lateral; $\boldsymbol{E}-\boldsymbol{F}: \mathrm{D}$, dorsal; $\mathrm{L}$, lateral.

rhinal fissure and overlaps with agranular cortex that lacks L4 and shows a much thinner $\mathrm{L} 5$ than in $\mathrm{M} 2^{+}$cortex (Fig. 1F). This cytoarchitecture suggest that the $\mathrm{M} 2^{-}$gap represents ECT (Dong, 2008) and that in coronal sections the gap corresponds to the M2 $2^{-}$band seen in tangential slices. Thus, agranular PORv lies in ECT and is not part of V1-recipient, $\mathrm{M}^{+}$postrhinal cortex.

\section{$\mathrm{M2}^{+}$patches in higher visual areas are linked to cortical magnification}

We have shown previously that alternating $\mathrm{M}^{+}$and $\mathrm{M}^{-}$ patches are not unique to $\mathrm{V} 1$, but is also observed throughout the thickness of L1, most notably at the L1/2 border in LM, LI, POR, PORa, auditory (AUD), and retrosplenial (RSP) cortex
(Ji et al., 2015). In addition, we noted that in each of these areas M2 expression is more uniform in layers below the L1/2 border (Fig. $2 A, B$ ). In $\mathrm{V} 1, \mathrm{M}^{+}$patches parcellate the plexiform $\mathrm{L} 1$ in the tangential plane into constant size domains, which extend to the cellular L2/3, and in groups of four to eight represent the visual point image (Ji et al., 2015). This organization raised the question of whether the shape, density, and cortical magnification factor of $\mathrm{M}^{+}$patches is related to the layout of the visuotopic maps in LM, LI, and POR. We determined the aspect ratio of $\mathrm{M}^{+}$patches along the major and minor axes of V1, LM, LI, and POR measured from the study by Garrett et al. (2014, their Fig. 7; see Materials and Methods). We found that in all four areas, average patch anisotropy was aligned with the axes of the 

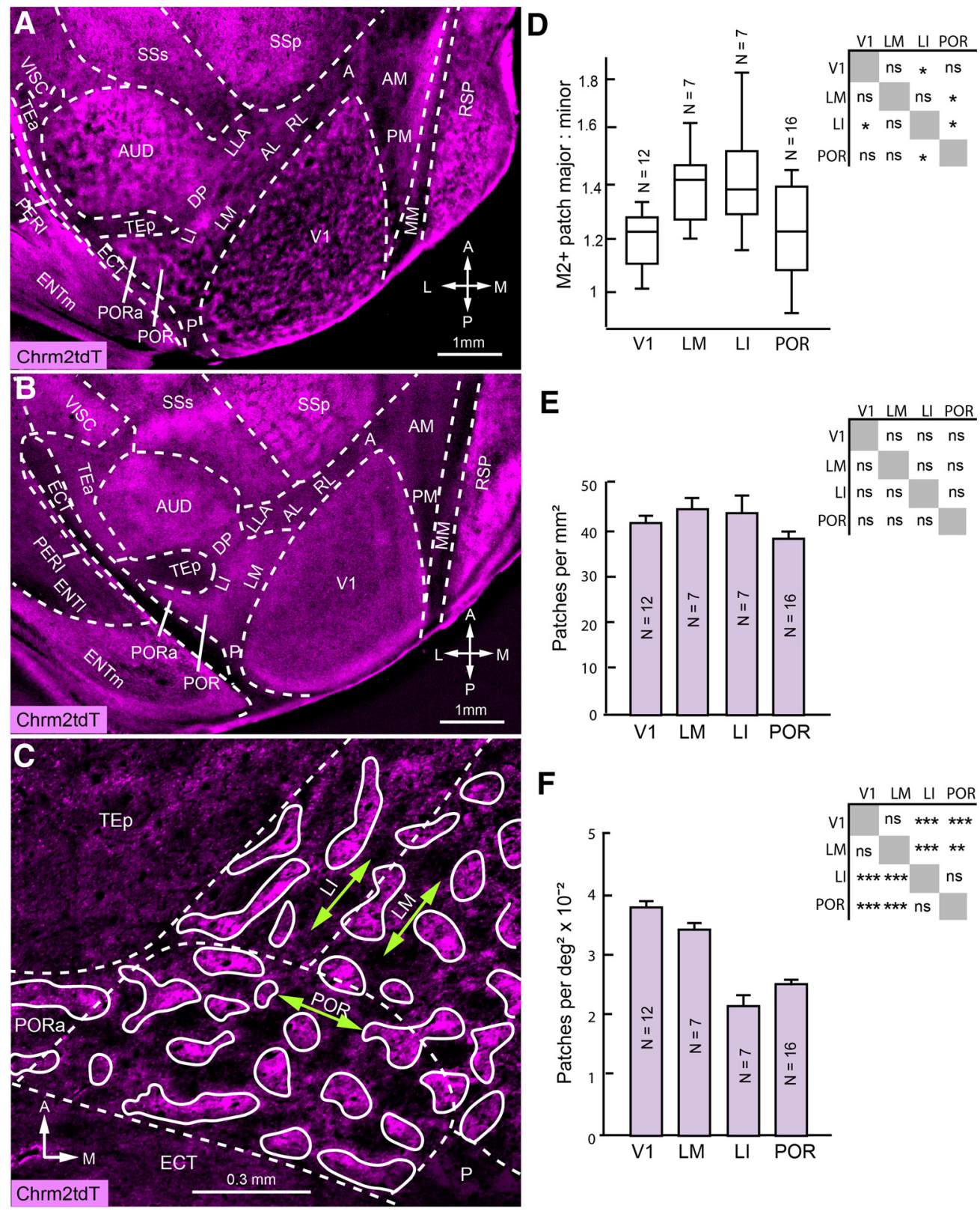

Figure 2. Patchy M2 expression in higher cortical areas. $A, B$, Tangential section through occipital cortex of Chrm2-tdT mouse shows patchy M2 expression throughout the thickness of $\mathrm{L} 1$ $(\boldsymbol{A})$ and uniform expression in L4 (B) of V1, LM, LI, POR, PORa, AUD, and RSP. Scale and orientation in $\boldsymbol{B}$ are the same as in $\boldsymbol{A}$. $\boldsymbol{C}$, Higher-magnification image of LM, LI, POR, and PORa shows that $\mathrm{M2}^{+}$patches are elongated parallel to the major axes (green double-headed arrows) of $\mathrm{LM}, \mathrm{LI}$, and POR. $\boldsymbol{D}$, Mean \pm SEM ratio of major/minor axial lengths of M2 ${ }^{+}$patches along the major axis of the containing area in V1, LM, LI, and POR (mean, 63.2 patches/mouse analyzed; Tukey's test). $E$, Mean \pm SEM spatial density of M2 ${ }^{+}$patches across areas (same mice and patches as in $\boldsymbol{D}$; Tukey's test). $\boldsymbol{F}$, Mean \pm SEM M2 ${ }^{+}$patches per square degree of visual space, determined by multiplying spatial densities with magnification factor of respective area (Garrett et al., 2014; same mice and patches as in $\boldsymbol{D}$; Tukey's test; $* p<0.05 ; * * p<0.01 ; * * * p<0.001$ ).

containing area (Fig. 2D). In areas V1, LI, and LM, whose major axes represent altitude, patches were stretched along elevation. In POR, with the major axis mapping azimuth, average patch elongation occurred along latitude. In V1, LM, and LI, patch dimensions closely matched those of their containing area [with major:minor length for patches; V1, $1.21 \pm 0.03$ (12 mice, 1354 patches); LM, 1:1.4 \pm 0.05 (7 mice, 122 patches); LI, $1: 1.42 \pm 0.08$ ( 7 mice, 78 patches); POR, $1.21 \pm 0.03$ (16 mice, 278 patches)] and areas (V1, 1:1.22; LM, 1:1.53; LI, 1:1.53; POR, 1: 1.59; Garrett et al., 2014). These results suggest that anisotropies in the representation of retinotopic space are shared by the $\mathrm{M}^{+}$patches within the areas we have examined.
Next, we determined the spatial distribution of $\mathrm{M}^{+}$patches and found that the density was similar in V1, LM, LI, and POR $(p>0.005$, Tukey's test; Fig. $2 E)$. These results indicate that the patch size (top $20 \%$ of $\mathrm{M}^{+}$pixels) is constant across areas, as was the case across different quadrants of V1 (Ji et al., 2015). How much of the visual field is represented by a $\mathrm{M} 2{ }^{+}$patch in a given area? To find out, we multiplied patch density (patches per square millimeter) by the cortical magnification factor (square millimeters per square degree; Garrett et al., 2014) and obtained a quantity patches per square millimeter in V1, LM, LI, and POR. We found that a $10 \times 10^{\circ}$-wide field of visual space, which accounts for about the size of the point image in V1 (12 mice) 

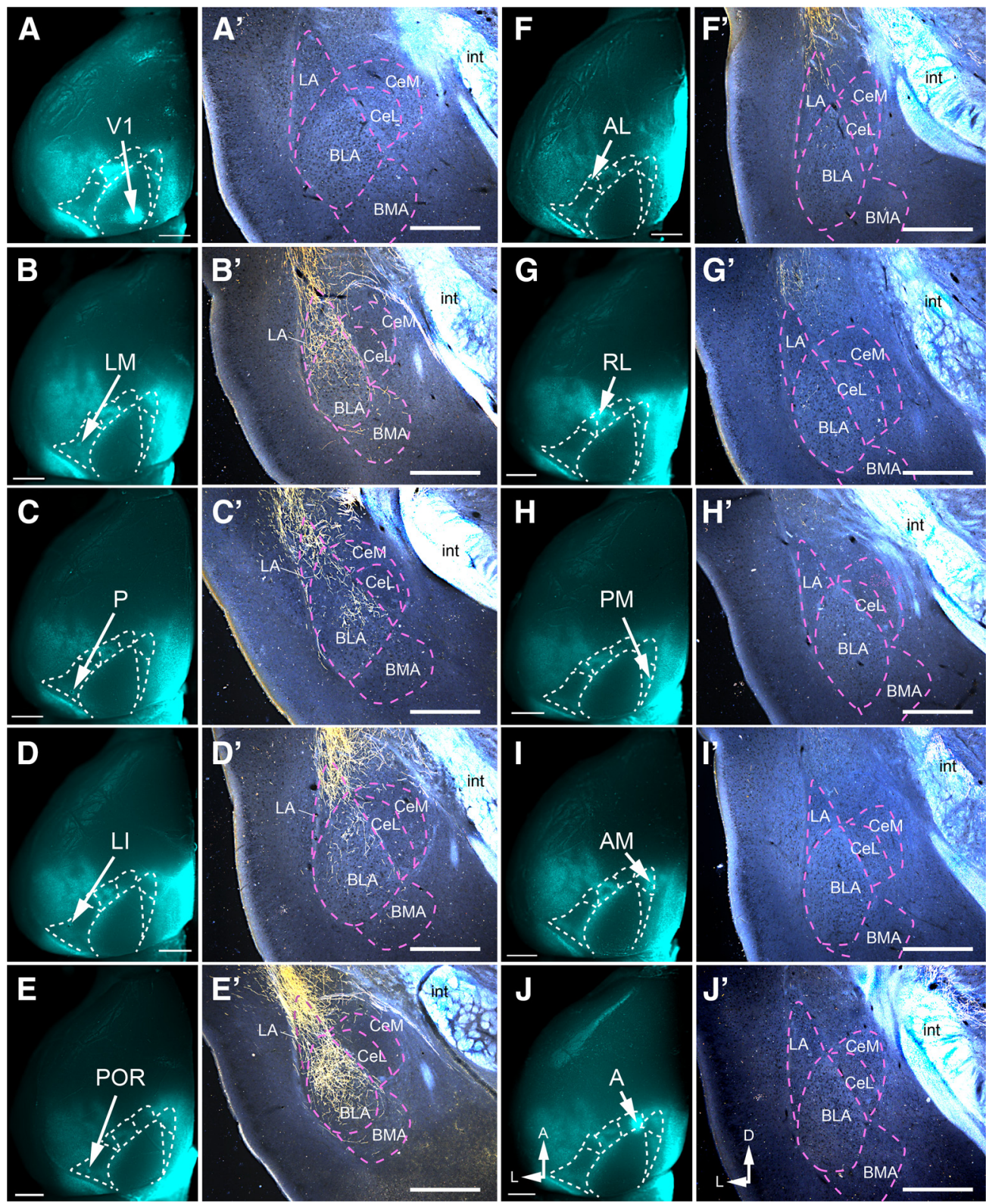

Figure 3. Axonal projections of visual cortex $\rightarrow$ amygdala pathways. A-J, In situ ex vivo images of callosal connections traced by retrograde transport of bisbenzimide from the posterior third of the right (contralateral) hemisphere. The arrows point to bright spots that represent the BDA injection sites in V1, LM, P, LI, POR, AL, RL, PM, AM, and A of the left (ipsilateral) hemisphere. $\boldsymbol{A}^{\prime}-\boldsymbol{J}^{\prime}$, Dark-field images of coronal sections showing anterogradely BDA-labeled axonal projections (yellow) from V1, ventral (LM, P, LI, POR), and dorsal (AL, RL, PM, AM, A) stream areas terminating in different nuclei of the amygdala. Note that the white structures represent unlabeled, highly reflective myelinated fibers of the internal capsule (int). Scale bars: $\boldsymbol{A}-\boldsymbol{J}$, $1 \mathrm{~mm} ; \boldsymbol{A}^{\prime}-J^{\prime}, 0.5 \mathrm{~mm}$.

and LM (7 mice; D'Souza et al., 2020), contains $3.93 \pm 0.08$ and $3.46 \pm 0.23$ patches $/ \mathrm{mm}^{2}$, respectively. In LI (7 mice) and POR (16 mice), a similar $10 \times 10^{\circ}$-wide field contained about half the number of patches, indicating that a single LI or POR patch represents a proportionately larger amount of visual space than a patch in V1 of LM ( $p<0.001$, Tukey's test; Fig. $2 F)$.

\section{Anterogradely labeled outputs from visual cortex to} amygdala, thalamus, brainstem, and striatum

Despite massive efforts, there are no detailed descriptions of the connectivity of visual cortical areas with the amygdala in mice, except for POR (Burgess et al., 2016). In previous studies (Oh et al., 2014; Zingg et al., 2014; Hintiryan et al., 2019), injections involved multiple areas, and areal assignments were based on the computed majority stake, which is known to underestimate the connectome density (Gămănuţ et al., 2018). We have taken a different approach in which we only accepted clean hits of the source area for anterograde tracing with BDA. To do this, we relied on the complementary patterns of M2 expression and callosal connections (Wang et al., 2011). Callosal connections were labeled by retrograde tracing with bisbenzimide and visualized in situ in fixed brains imaged under a fluorescence stereomicroscope. This procedure also revealed the injection site as a bright spot, which was, based on the distinctive callosal projection pattern, readily assigned to V1, PM (posteromedial), AM (anteromedial), A (anterior), RL (rostromedial), AL (anterolateral), LM, LI, P, or POR (Wang and Burkhalter, 2007; Fig. 3A-J). The cortex dorsal to the rhinal fissure was then separated from the 
brain, flattened, cut tangentially, reacted for M2 immunofluorescence, and stained with an $\mathrm{ABC}$ reaction to reveal the $\mathrm{BDA}$ injection site. Injections were considered clean hits if they were confined within the areal boundaries delineated by Gămănut et al. (2018). The rest of the brain was cut coronally, and sections containing the amygdala were stained to reveal BDA-labeled axonal projections. We found that the projections from $\mathrm{V} 1$ and the dorsal stream areas, $\mathrm{AL}, \mathrm{RL}, \mathrm{PM}, \mathrm{AM}$, and $\mathrm{A}$, were extremely weak and too sparse for determining their optical density (Fig. $3 F^{\prime}-$ $\left.J^{\prime}\right)$. In sharp contrast, the projections from the ventral stream areas, LM, P, LI, and POR, were much denser (Fig. $\left.3 A^{\prime}-E^{\prime}\right)$. The vast majority $(98-100 \%)$ of inputs from LM, $\mathrm{LI}$, and $\mathrm{P}$ to the amygdala terminated in LA with only minor offshoots to BLA (basolateral amygdala; Fig. $3 B^{\prime}-D^{\prime}$ ). Projections from POR were percentage-wise (percentage of total input to amygdala and percentage of optical density relative to injection site) the strongest in LA, but, compared with LM, LI, and $\mathrm{P}$, a larger fraction terminated in BLA and central amygdala (CeA; Fig. 4B-E, inset).

Compared with the total output from 10 visual cortical areas to 31 subcortical targets, the weight of amygdala projections was weak, and even the strongest combined input from POR to LA and BLA was only $\sim 10 \%$ of the total strength (Fig. $4 A-J$ ). Notably, inputs from ventral areas to LA were significantly stronger than from dorsal areas (Fig. $4 K)$. POR input to another emotion regulatory center for fear, the dorsal lateral periaqueductal gray (DLPAG), which responds to cortical stressors and, unlike the amygdala, does not receive visceral input (Floyd et al., 2000), was only $\sim 2 \%$ (Fig. 4E). Similarly sparse projections to DLPAG were found from P, AM, and PM (Fig. 4D,H,I).

Bennett et al. (2019) have shown that dorsal stream areas are more weakly connected to posterior LP than areas of the ventral stream. We found a similar rostrocaudal distribution by anterograde BDA labeling of outputs from nine higher visual areas. In our data, however, ventral stream projections include the rostral LP, the primary target of the dorsal stream. The strong preference for posterior LP [LP, laterocaudal (LPLC); LP, mediocaudal (LPMC)] was present in the inputs from ventral stream areas LM, LI, P, and POR (Fig. $4 B-E$ ), which was in striking contrast to the absence of input from the dorsal stream, AL, RL, AM, PM, and A (Fig. 4F-J). Inputs to the middle and anterior part of LP [LP, laterorostral (LPLR); LP, mediorostral (LPMR)], including the widely connected laterodorsal nucleus, dorsomedial, and laterodorsal nucleus, ventrolateral (laterodorsal thalamic nuclei; Juavinett et al., 2020), originated from both ventral and dorsal stream areas (Fig. 4B-J). Unexpectedly, we found that POR sent weak outputs to dLGN, from which it also received weak inputs (Figs. $4 E, 5 D, E$ ). Notably all of the retrogradely DY-labeled cells found in the dLGN were located in the shell (Fig. 5D,E). This suggests that intracortical communication through transthalamic circuits (Sherman, 2016) may involve the higher-order LP as well as the first-order dLGN. Weak POR projections were also found to the auditory medial geniculate, ventral (MGV), and marginal zone of medial geniculate (Fig. 4E). Unlike dorsal stream areas, which more strongly project to oculomotor and motor coordination centers than ventral stream areas, such inputs were extremely weak or absent from POR (Fig. 4E). A notable exception was the strong POR inputs to the dorsomedial caudoputamen (Fig. 4E), which plays a role in goal-directed behavior (Hunnicutt et al., 2016).

\section{Retrogradely labeled sources of inputs to POR}

To map the sources of inputs to POR, we retrogradely traced neurons in PVtdT mice with DY $(N=2)$. Unsurprisingly, given the results from anterograde tracing (Fig. $4 E$ ), we found DY-labeled cells in LA, LP, and dLGN (Fig. 5D-G). Of a total of 2454 DY-labeled neurons in the primary and secondary visual thalamus, the vast majority were found in different subdivisions of

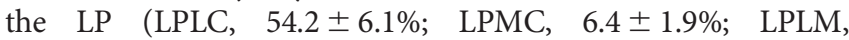
$25.3 \pm 3.4 \%$; LPMR, $11.7 \pm 2.0 \%)$. Only a small percentage of labeled cells was encountered in the dLGN (2.4 $\pm 1.8 \%)$, notably all of them in the dLGN shell (Fig. 5D,E). This shows that POR receives retinal input via a direct $\mathrm{dLGN} \rightarrow \mathrm{POR}$ and an indirect $\mathrm{SC} \rightarrow \mathrm{LP} \rightarrow$ POR (Beltramo and Scanziani, 2019) thalamocortical pathway. In addition, we found DY-labeled POR projecting thalamic neurons in the zona incerta, suprageniculate, dorsal anteromedial, central medial thalamic nucleus, paratenial thalamic nucleus, and MGV (data not shown). Limbic regions contained DY-labeled cells in LA; medial (ENTm) and lateral (ENTl) entorhinal cortex (Fig. 5B,C,G). Each of these structures received strong inputs from POR (Figs. $3 E^{\prime}, 4 E^{\prime}$; Wang et al., 2012), suggesting bidirectional communications among POR, amygdala (confirming the findings of Burgess et al., 2016), and entorhinal cortex (Fig. 6A-C; also see Figs. 8A-C,E-G, 9A-C,E$G)$. DY-labeled POR projecting neurons were also encountered in multiple neocortical areas including the following: V1; PM; MM (mediomedial area), RSP (retrosplenial area); AM; RL; AL; LM; LI; P; ECT; TEp; TEa (anterior temporal area); DP (dorsal posterior area), AUD, AIv (ventral anterior insula); GU (gustatory area), ORBl (lateral orbitofrontal area), ILA (anterior infralimbic area), and ACAv (ventral anterior cingulate area) (Fig. 5A-C).

\section{Anterogradely labeled projections from amygdala to POR terminate in $\mathrm{M}^{-}$interpatches}

It has been known from studies in rats and mice that projections from LA and BLA to POR terminate in L1-3 with weaker input to the layers below (Pikkarainen and Pitkänen, 2001; Burgess et al., 2016). To find out whether these inputs are modularly organized, we traced inputs from LA and BLA (Fig. 6B, inset) with AAV.EGFP or AAV.tdT in C57BL/6 and Chrm2-tdTmice. We found that the laminar distribution of inputs from both sources was similar, which let us pool (LA/BLA, $N=5$ ) the baseline-subtracted fluorescence intensity of axonal projections. Inputs were densest in L1, weaker in L3/4, and sparse in L2, L5, and L6 (Fig. $7 E)$. To study whether inputs specifically target $\mathrm{M}^{+}$patches or $\mathrm{M} 2^{-}$interpatches, we removed the cortex dorsal to the rhinal fissure, flatmounted the tissue, cut tangential sections, and sliced the amygdala in the standard coronal plane. Projections to L1 of POR were strikingly nonuniform, preferentially targeted $\mathrm{M}^{-}$ interpatches, and largely avoided $\mathrm{M}^{+}$patches (Fig. 6A-C). We performed an automated delineation of $\mathrm{M}^{+}$patch borders with custom MATLAB scripts, using a procedure similar to that used by Sincich and Horton (2005). M2 intensity images were highpass filtered and blurred, then partitioned into six quantiles based on fluorescence intensity (Fig. $6 D, E$ ). To test for different input strengths to patches and interpatches, EGFP intensity was compared across M2-defined quantiles (Fig. 6F). EGFP intensity showed a strong negative correlation with M2 expression $(R=$ $-0.86, p<10^{-8}, N=5$, Pearson correlation), and projection strength in the highest quantile (6) was a mere $17 \%$ of that in the lowest quantile (1), indicating that $\mathrm{M} 2^{-}$interpatches received significantly stronger input from the LA/BLA than $\mathrm{M}^{+}$patches (Figs. 6F, $7 F$ ). 

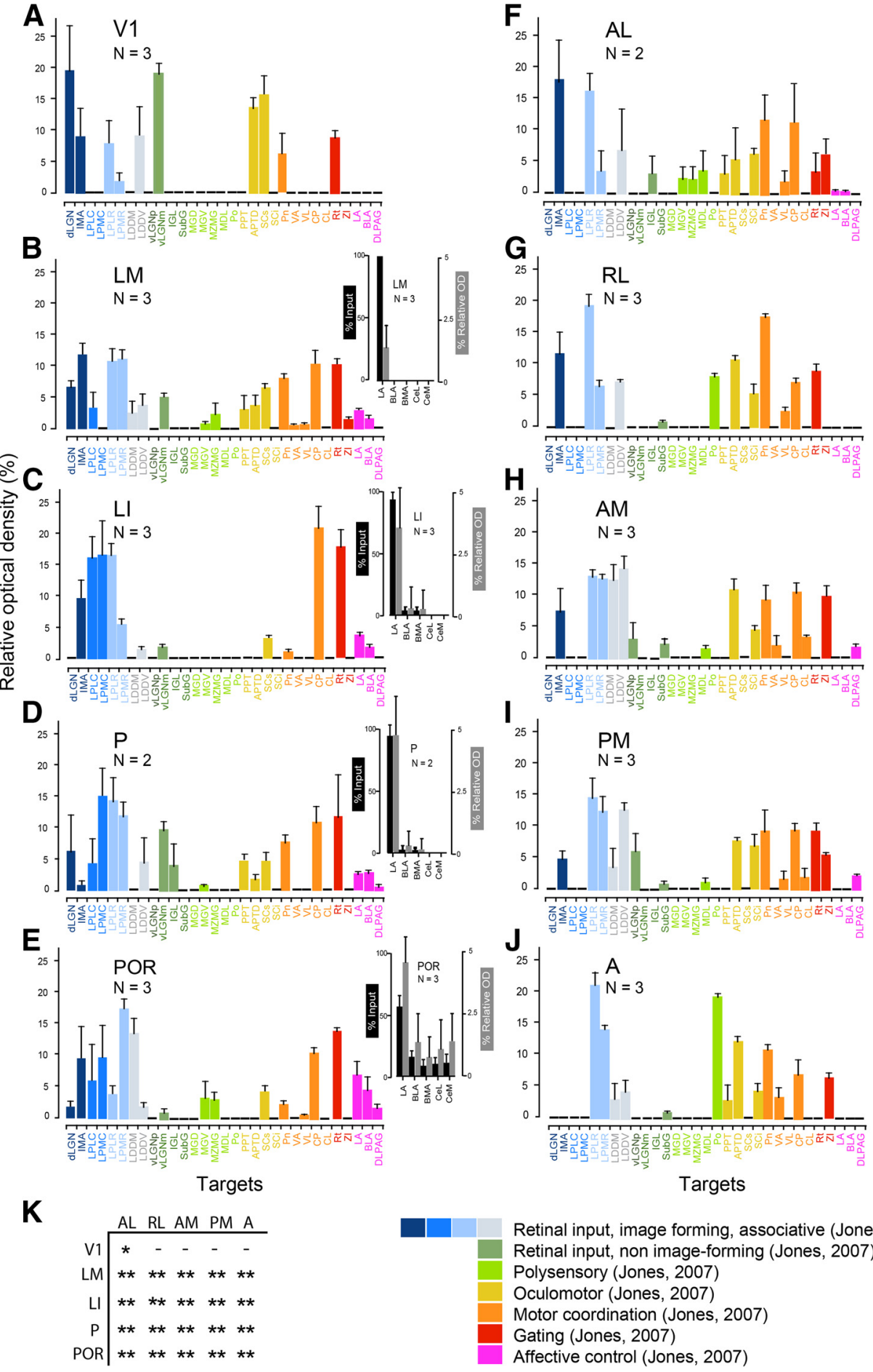

Retinal input, image forming, associative (Jones, 2007)

Retinal input, non image-forming (Jones, 2007)

Polysensory (Jones, 2007)

Oculomotor (Jones, 2007)

Motor coordination (Jones, 2007)

Gating (Jones, 2007)

Affective control (Jones, 2007)

Figure 4. Relative strength of anterogradely BDA-labeled projections of mouse visual cortex to 31 subcortical targets. Nomenclature is after that in Dong (2008) and Franklin and Paxinos (2008). A-E, Average \pm SEM optical density of projections from V1 and the ventral stream areas LM, LI, P, and POR in percentage of the summed density of all subcortical projections labeled from each area. $\boldsymbol{F}$-J, Projections from the dorsal stream areas AL, RL, AM, PM, and A. Colors indicate functionally broadly similar groups of subcortical targets (Jones, 2007). $\boldsymbol{B}-\boldsymbol{E}$, insets, Relative strength of anterogradely BDA-labeled projections from ventral stream areas LM, LI, P, and POR to LA, BLA, BMA, CeL, and CeM. Percentage (mean \pm SEM) of total optical density of labeled terminal axonal branches (black bars). Values are background-subtracted percentage (mean \pm SEM) of optical density relative to the injection site (gray bars). $\boldsymbol{K}$, Comparison of projection strengths from different visual areas to LA. Two-sample $t$ test: $* p<0.001 ; * * p<0.0001$. (-), undetectable; (eL, centrolateral amygdala; CeM, centromedial amygdala).

\section{Anterogradely labeled projections from dLGN and LP to POR terminate in $\mathrm{M2}^{+}$patches}

We have found recently that M2 expression in V1 divides L1 into interdigitating modules receiving dLGN input to $\mathrm{M}^{+}$ patches and LP input to $\mathrm{M}^{-}$interpatches (Ji et al., 2015;
D'Souza et al., 2019). Both of these thalamic nuclei are also known to project to extrastriate visual cortex (Oh et al., 2014), but only LP has been shown unequivocally to project to POR (Zhou et al., 2017; Beltramo and Scanziani, 2019; Bennett et al., 2019; Juavinett et al., 2020). Here, we investigated whether 

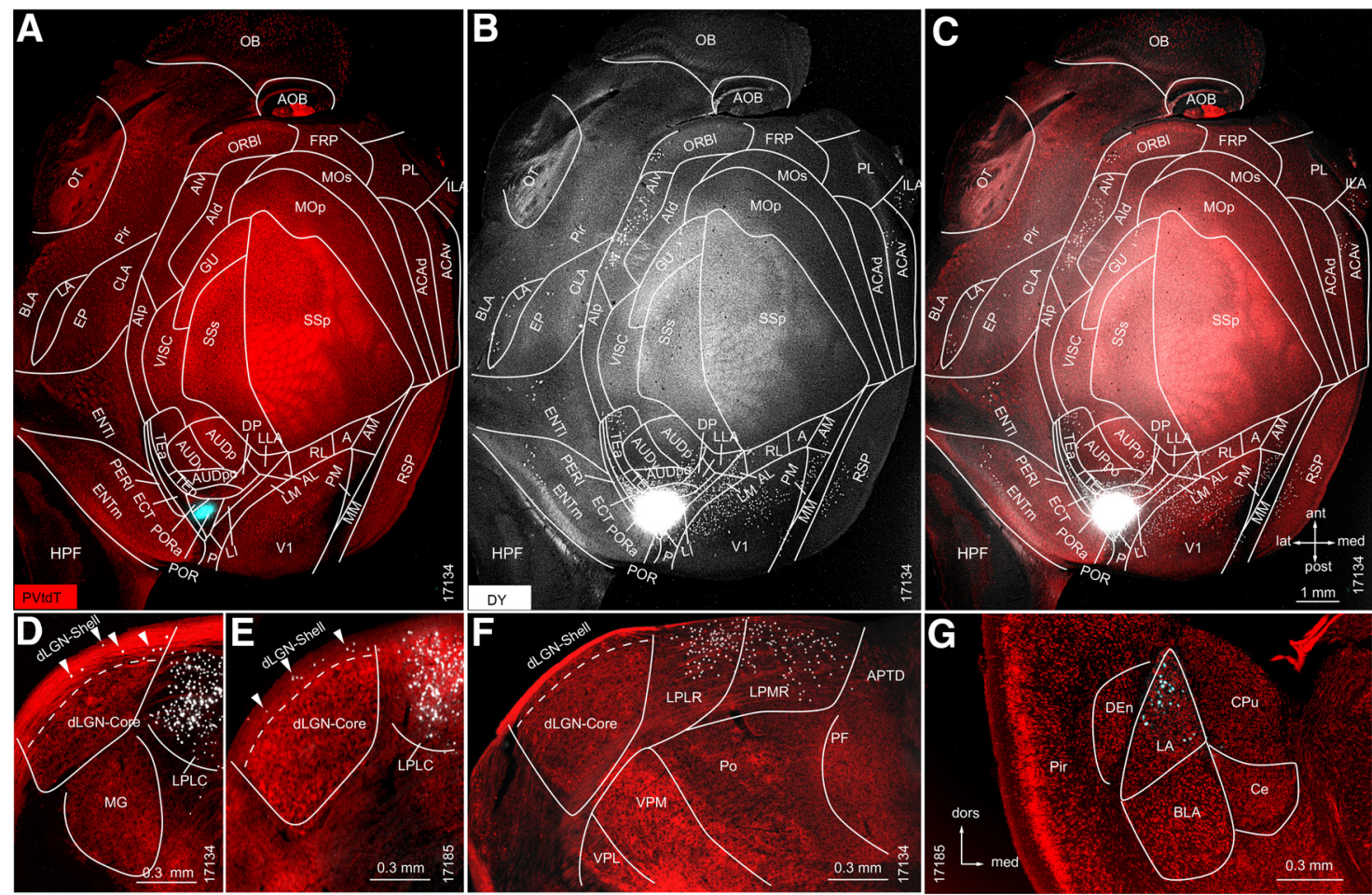

Figure 5. Retrograde tracing in PVtdT mice of subcortical and cortical source neurons projecting to POR. $\boldsymbol{A}$, Tangential section through L2/3/4 of flatmounted PVtdT-expressing (red) cortex, shows DY injection site (false colored blue) in POR. B, Overexposed black/white image (notice the artificially large injection site gives the false impression that DY spilled beyond the borders of POR) showing DY-labeled neurons (white dots) in LA and multiple cortical areas segmented according to Gămănuţ et al. (2018). C, Overlay of DY-labeled neurons with PVtdT expression. D-G, Coronal sections through thalamus and amygdala. $\boldsymbol{D}, \boldsymbol{E}$, Representative sections through the dLGN of two different mice (17134 and 17185) showing that DY-labeled neurons are present in the shell (arrowheads) but not in the core. $\boldsymbol{F}, \mathbf{G}$, Dense labeling in the LPLR and LPMR $(\boldsymbol{F})$, and LA (G).

dLGN and LP project to POR and whether inputs to L1 show an interdigitating pattern similar to the one we have found in V1 (D'Souza et al., 2019). For this purpose, we injected the anterograde viral tracers (AAV.EGFP, AAV.tdT) into either dLGN or LP (Fig. $7 B, D$, inset). We found that input from dLGN $(N=5)$ and LP $(N=2)$ terminated in POR and PORa (Fig. $7 A-D)$. In two cases with a complete series of sections across cortex, we found that dLGN input was densest in L1 and L5, moderate in L4 and L6, and weakest in L2/3 (Fig. 7E). LP input was dense in L1 and L4, moderate in L5 and L6, and weakest in L2/3 (Fig. 7E). To determine the patch/interpatch ratio of input to $\mathrm{L} 1$, the top three M2 quantiles were taken as $\mathrm{M}^{+}$patches and the bottom three quantiles as $\mathrm{M}^{-}$interpatches. The results show that the tangential distribution of dLGN and LP inputs to L1 of POR and PORa overlapped with $\mathrm{M}^{+}$patches (patch/interpatch ratios: $\mathrm{dLGN} \rightarrow$ POR, $3.04 \pm 0.67, N=5, p<0.01$, $t$ test; LP $\rightarrow$ POR, $3.51 \pm 0.42, N=2, p<0.01, t$ test; Fig. $7 F)$. The targeting in POR and PORa to $\mathrm{M}^{+}$patches distinguished dLGN and LP input from amygdala inputs, which terminated in $\mathrm{M}^{-}$interpatches (Fig. 6C,F). EGFP intensity positively correlated with M2 quantile $(\mathrm{LP} \rightarrow$ POR, $p<0.001, r=0.83, N=$ mice; $d L G N \rightarrow$ POR, $p<10^{-11}, r=0.91, N=5$ mice, Pearson correlation). dLGN and LP inputs to LM and LI likewise targeted $\mathrm{M}^{+}$patches and avoided $\mathrm{M}^{-}$interpatches (Fig. 7F; patch/interpatch ratios: $\mathrm{dLGN} \rightarrow \mathrm{LM}, 2.45 \pm 0.59, N=4, p<0.01, t$ test; LP $\rightarrow$ LM, $2.11 \pm 0.71, N=3, p<0.05, t$ test; dLGN $\rightarrow$ LI, $2.09 \pm 0.49$, $N=4, p<0.01, t$ test; LP $\rightarrow$ LI, $2.1 \pm 0.75, N=3, p<0.05, t$ test). In agreement with previous studies (D'Souza et al., 2019), dLGN inputs to $\mathrm{V} 1$ were preferentially targeted to $\mathrm{M}^{+}$patches, whereas LP inputs preferred $\mathrm{M}^{-}$interpatches (data not shown). Inputs from $\mathrm{V} 1 \rightarrow \mathrm{POR}$, while topographical (Fig. 1B,C), showed no clear preference for either compartment (patch/interpatch ratio: $1 \pm 0.03, N=4, p=0.88, t$ test; Fig. $7 F$ ).

\section{Cell bodies and apical dendrites of POR $\rightarrow$ amygdala and $\mathrm{POR} \rightarrow \mathrm{ENTm}$ projecting neurons are aligned with $\mathrm{M}^{-}$interpatches}

The striking specificity of amygdala $\rightarrow$ POR axonal projections for $\mathrm{M}^{-}$interpatches suggested that these inputs may target apical dendrites of long-range projecting neurons in L1. Of particular interest were POR neurons projecting to downstream targets such as the amygdala and ENTm, which are known for their roles in affective processing (Phelps and LeDoux, 2005), memory, and spatial navigation (Buzsáki and Moser, 2013), respectively. To label the complete dendritic arbor of such neurons, we performed retrograde viral tracing from LA/BLA and ENTm in Ai9 mice with AAV2retro-CAG-Cre. $\mathrm{M}_{2}{ }^{+}$patches were identified by immunostaining for M2 and dividing fluorescence intensity into quantiles. The results show that LA/BLA and ENTm injections retrogradely labeled pyramidal cell dendrites, somata, and occasionally their local axonal projections in POR (Fig. 8AG), PORa, P, ECT, LM, and LI (Fig. 8I,J).

Dendrites were readily distinguished from axons by the greater thickness and positively identified based on the presence of spines (Vaughan and Peters, 1973; Karimi et al., 2020). Module preference of apical dendrites in L1 was quantified by reconstructing branches in three to four ROIs of the top two sections through POR. An example is shown in Figure $8 D$. Most strikingly, we found that apical dendrites of POR $\rightarrow$ LA/BLA projecting cells branched preferentially in $\mathrm{M}^{-}$interpatches of L1 (Fig. $8 E-G$ ). Similar results were obtained for $\mathrm{POR} \rightarrow$ ENTm projecting neurons 

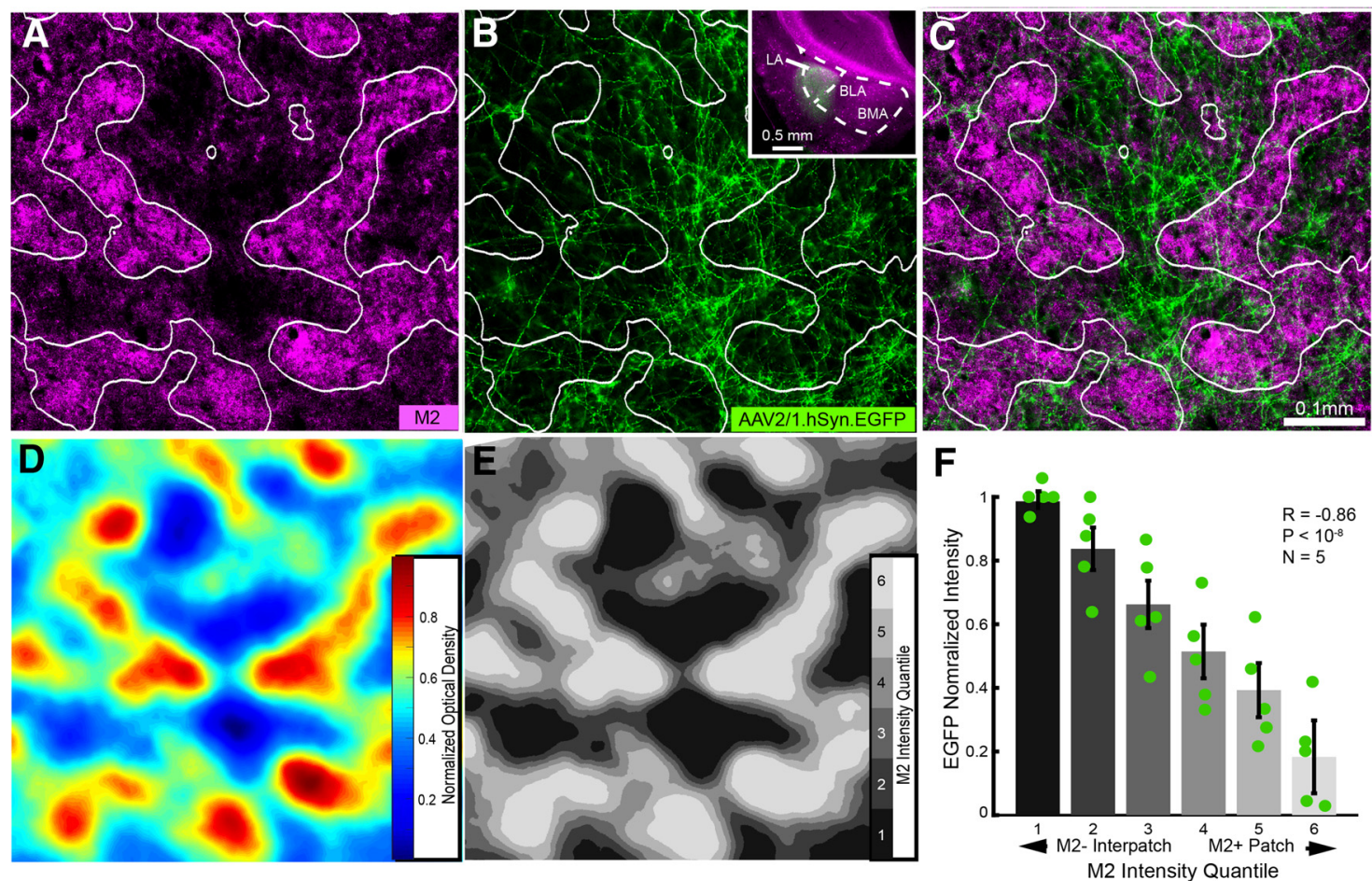

Figure 6. Axonal projections of lateral amygdala $\rightarrow$ POR pathway. $A$, Tangential section through $\mathrm{L} 1$ of flatmounted POR in Chrm2-tdT mouse showing nonuniform pattern of $\mathrm{M2} 2^{+}$patches (magenta) and M2 ${ }^{-}$interpatches (black). $\boldsymbol{B}$, Patchy axonal projections to L1 labeled by anterograde tracing with AAV2/1.hSyn.EGFP from the LA. Coronal section of injection site in LA ( $\boldsymbol{B}$, inset). $\boldsymbol{C}$, Overlay of $\boldsymbol{A}$ and $\boldsymbol{B}$ shows that $\mathrm{LA} \rightarrow$ POR projection to $\mathrm{L} 1$ terminates preferentially in $\mathrm{ML}^{-}$interpatches. $\boldsymbol{D}$, Heat map of M2 expression in POR $(\boldsymbol{A})$. $\boldsymbol{E}$, Partitioning of heat map $(\boldsymbol{D})$ into six equal-area quantiles (different shades of gray) for the delineation of $\mathrm{ML}^{+}$patches and $\mathrm{ML}^{-}$interpatches. $\boldsymbol{F}$, Normalized average \pm SEM $(N=5$ mice) EGFP fluorescence intensity in each of the six quantiles shows that $\mathrm{LA} \rightarrow \mathrm{POR}$ inputs are preferentially associated with $\mathrm{M} 2^{-}$interpatches $\left(R=-0.86, p<10^{-8}\right.$, Pearson correlation).

(Fig. 8A-D). Comparison of total dendrite lengths in M2 intensity quantiles showed a strong negative correlation between M2 intensity and labeled dendrites (POR $\rightarrow$ LA/BLA: $R=-0.93, p<10^{-10}$, $N=4$ mice; POR $\rightarrow$ ENTm: $R=-0.99, p<10^{-8}, N=4$ mice, Pearson correlation), supporting the finding of spatial clustering in M2 ${ }^{-}$interpatches (Fig. $8 H$ ).

In cases in which complete $z$-stacks of sections were available, somas and retrogradely labeled POR $\rightarrow$ ENTm $(N=2)$ and POR $\rightarrow$ LA/BLA $(N=2)$ cells were found in L26 of POR (Fig. 8I,J). Similar size injections into ENTm and LA consistently labeled more cells in POR than in any other area. LM $\rightarrow$ ENTm and LI $\rightarrow$ ENTm neurons were mostly confined to L2/3. P $\rightarrow$ ENTm, PORa $\rightarrow$ ENTm, and POR $\rightarrow$ ENTm cells were biased to deep layers with a preference for the L5/6 border (Fig. 8I). The ratio of total labeled POR $\rightarrow$ ENTm L2/3 cells to L5/6 cells within a given case was $1: 4.8 \pm 2.5(N=2, p<0.042, t$ test $)$. Deep layers were also the preferred source of cells projecting to LA/BLA (L2/3-toL5/ 6 ratio, $1: 2.8 \pm 0.1\left(N=2, p<10^{-3}, t\right.$ test $)$.

Unlike POR $\rightarrow$ ENTm cells, POR $\rightarrow$ LA/BLA neurons were distributed across the thickness of L5 (Fig. 8I,J), suggesting that the projections originate from distinct cell types.

We quantified the alignment of retrogradely labeled L2/3 neurons (sections across L4-6 were not available for analysis) with $\mathrm{M} 2^{+}$ patches and $\mathrm{M}^{-}$interpatches by overlaying M2 expression images with labeled cells and determining cell counts in each of six M2 quantiles. Cell counts were normalized by dividing the cell count within each quantile by the total number of labeled cells in that image. POR $\rightarrow$ ENTm cells showed a strong preference for aligning with $\mathrm{M}^{-}$interpatches (Fig. $9 A-D ; R=-0.86, p<10^{-5}, N=4$ mice, 132 total cells, Pearson correlation). POR $\rightarrow$ LA/BLA cells also showed a significant preference for aligning with $\mathrm{M}_{2}^{-}$ interpatches, though with less specificity than POR $\rightarrow$ ENTm cells (Fig. 9E-H; $R=-0.63, p<0.027, N=2$ mice, 53 cells, Pearson correlation).

Input from POR to hippocampus strongly biased to dorsal CA1 We have shown previously that POR provides input to CA1 of the hippocampal formation (HPF) but have not described the topographic organization of this pathway (Wang et al., 2012). Here, we have retraced the connections anterogradely with AAV2/1.hSyn.EGFP from POR in two mice. The case illustrated in Figure 10 shows a tangential section through flatmounted cortex. The injection site was assigned to POR by location and clearly spares PORa (Fig. 10A). To physically flatmount the HPF (for complete graphical flatmounting, see Swanson and Hahn, 2020), it was separated from the external capsule along the alveus, flipped backward so that the dorsomedial pole came to lie at the posterior edge of the section (Fig. 10B, inset). The green-labeled axonal projection was densest in the dorsal part of CA1 and gradually lost strength toward the ventral pole (Fig. 10B). A similar nonuniform distribution of projections was found when the injection involved POR and PORa, but was confined to the $\mathrm{M}^{+}$region (data not shown). This suggests that either the projections from POR and PORa are similar or that input from PORa was undetectable. Regardless, the results show that POR input is strongly biased to the dorsomedial part of the HPF. The results further show that POR $\rightarrow \mathrm{CA} 1$ input can either be direct, bypassing ENTm, or indirect, by way of ENTm (Wang et al., 2012). Both of these pathways provide visuospatial information (Fanselow and Dong, 2010), which through the reciprocal PORamygdala network (Figs. 6, $8 E-H$ ) receives affective input to influence spatial navigation. 


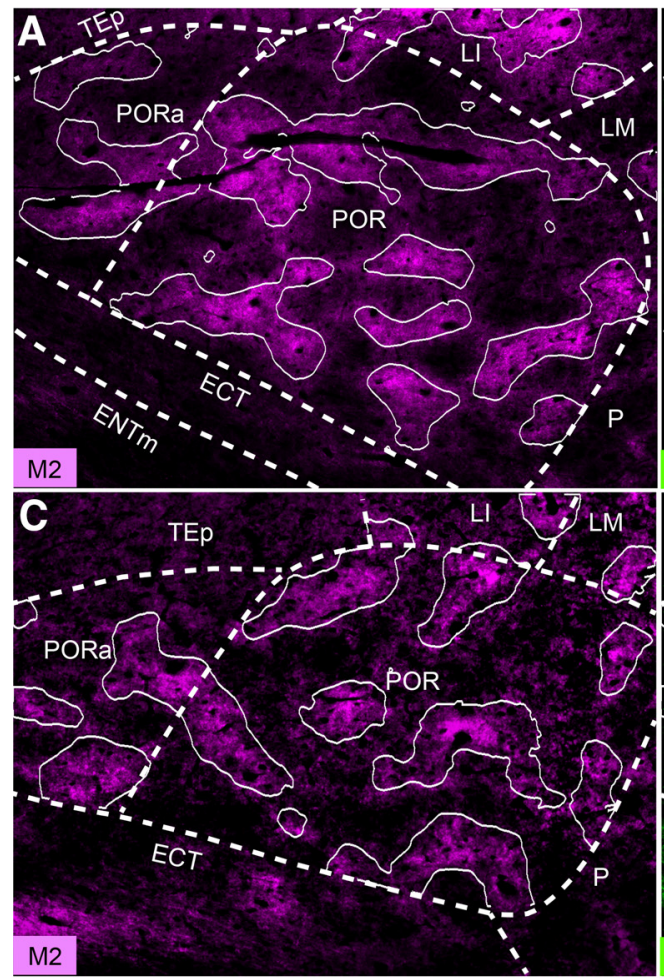

E

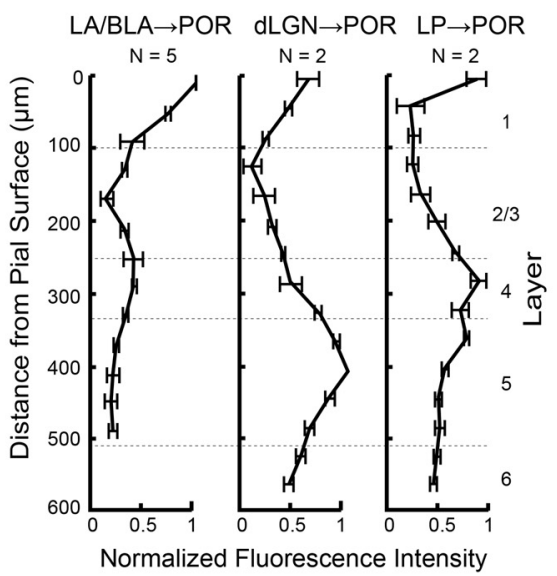

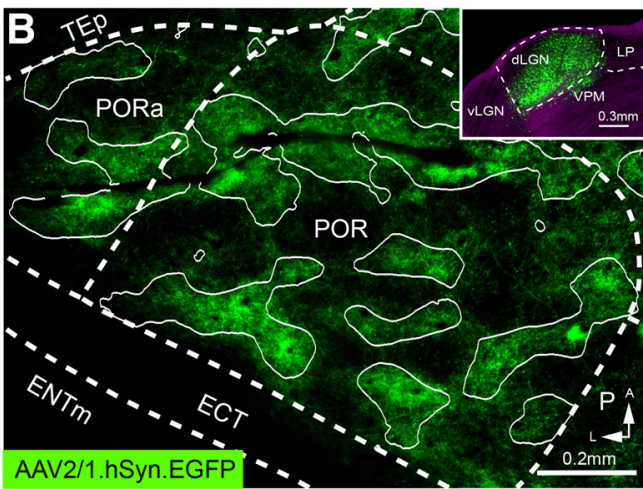

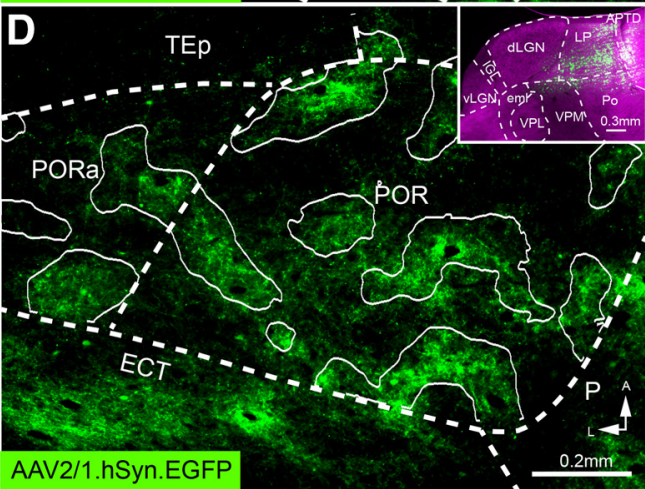

$\mathbf{F}$

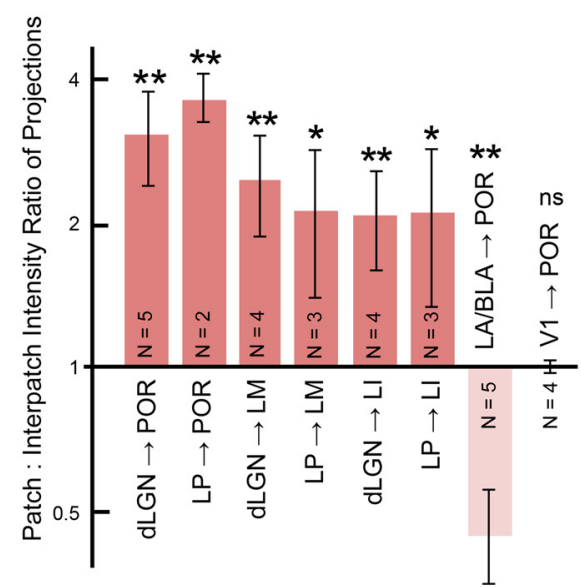

Figure 7. Axonal projections of $\mathrm{dLGN} \rightarrow \mathrm{POR}$ and $\mathrm{LP} \rightarrow \mathrm{POR}$ pathways. $\boldsymbol{A}, \boldsymbol{C}$, Immunostained $\mathrm{M2}^{+}$patches in $\mathrm{L} 1$ of flatmounted tangential section through POR and PORa. $B$, dLGN $\rightarrow$ POR axonal projections traced anterogradely with AAV2/1.hSyn.EGFP. $\boldsymbol{B}$, inset, Coronal section of injection site spreading across core and shell of dLGN. White contours indicate borders between fourth and fifth intensity quantiles (of six) of M2 expression (A). Overlay of contours shows overlapping patterns of $\mathrm{M}^{+}$patches and dLGN $\rightarrow$ POR projections $(\boldsymbol{B})$. $\boldsymbol{D}$, AAV2/1.hSyn.EGFP-labeled LP $\rightarrow$ POR projections to $\mathrm{ML}^{+}$patches $(\boldsymbol{C})$ in L1 of POR. $\boldsymbol{D}$, inset, Coronal section of injection site in LP. $\boldsymbol{E}$, Laminar distribution of anterogradely labeled LA/BLA $\rightarrow$ POR, dLGN $\rightarrow$ POR, and LP $\rightarrow$ POR projections. $\boldsymbol{F}$, Mean \pm SEM patch: interpatch EGFP intensity ratio of dLGN $\rightarrow$ POR, LP $\rightarrow$ POR, dLGN $\rightarrow \mathrm{LM}, \mathrm{LP} \rightarrow \mathrm{LM}, \mathrm{dLGN} \rightarrow \mathrm{LI}, \mathrm{LP} \rightarrow \mathrm{LI}, \mathrm{LA} / \mathrm{BLA} \rightarrow \mathrm{POR}$, and V1 $\rightarrow$ POR axonal labeling in $\mathrm{L} 1$ of the projection target. $* * p<0.01 ; * p<0.05$; ns, not significant (one-sample $t$ test).

\section{Discussion}

\section{Postrhinal cortex contains two modularly organized areas}

We have found that postrhinal cortex contains two separate areas, POR and PORa, with distinct visuotopic maps and connection profiles (Gămănuţ et al., 2018). Both areas have granular cytoarchitectures, are contained within an $\mathrm{M}^{+}$region, and have borders with the surrounding $\mathrm{M}^{-}$areas of ECT and TEp. We found no support for a ventral PORv (Beaudin et al., 2013), which based on its agranular cytoarchitecture and lack of M2 expression may correspond to rodent ECT or primate parahippocampal cortex TH (Burwell, 2001). The POR/PORa border was revealed by a transition from the orderly map in POR to the more dispersed but spatially clustered connectivity in PORa. A similar map reversal exists between primate parahippocampal cortices TFO and TF (Ungerleider et al., 2008), suggesting that the areal organization of the PV-and M2-expressing parahippocampal cortex (Saleem et al., 2007) in rodents and primates may be homologous.

A striking feature of POR, PORa, LM, and LI is the nonuniform tangential distribution of $\mathrm{M}^{+}$patches and $\mathrm{M}^{-}$interpatches in L1. Patchiness is a known attribute of primate extrastriate cortex (Kaas, 2012) and has also been shown to exist in the intrinsic connectivity of TF (Lavenex et al., 2004). The patchiness in POR, PORa, LM, and LI resembles the pattern we have seen in mouse V1 (Ji et al., 2015). However, unlike the quasi-isotropic modules in $\mathrm{V} 1$, patches in higher visual areas 

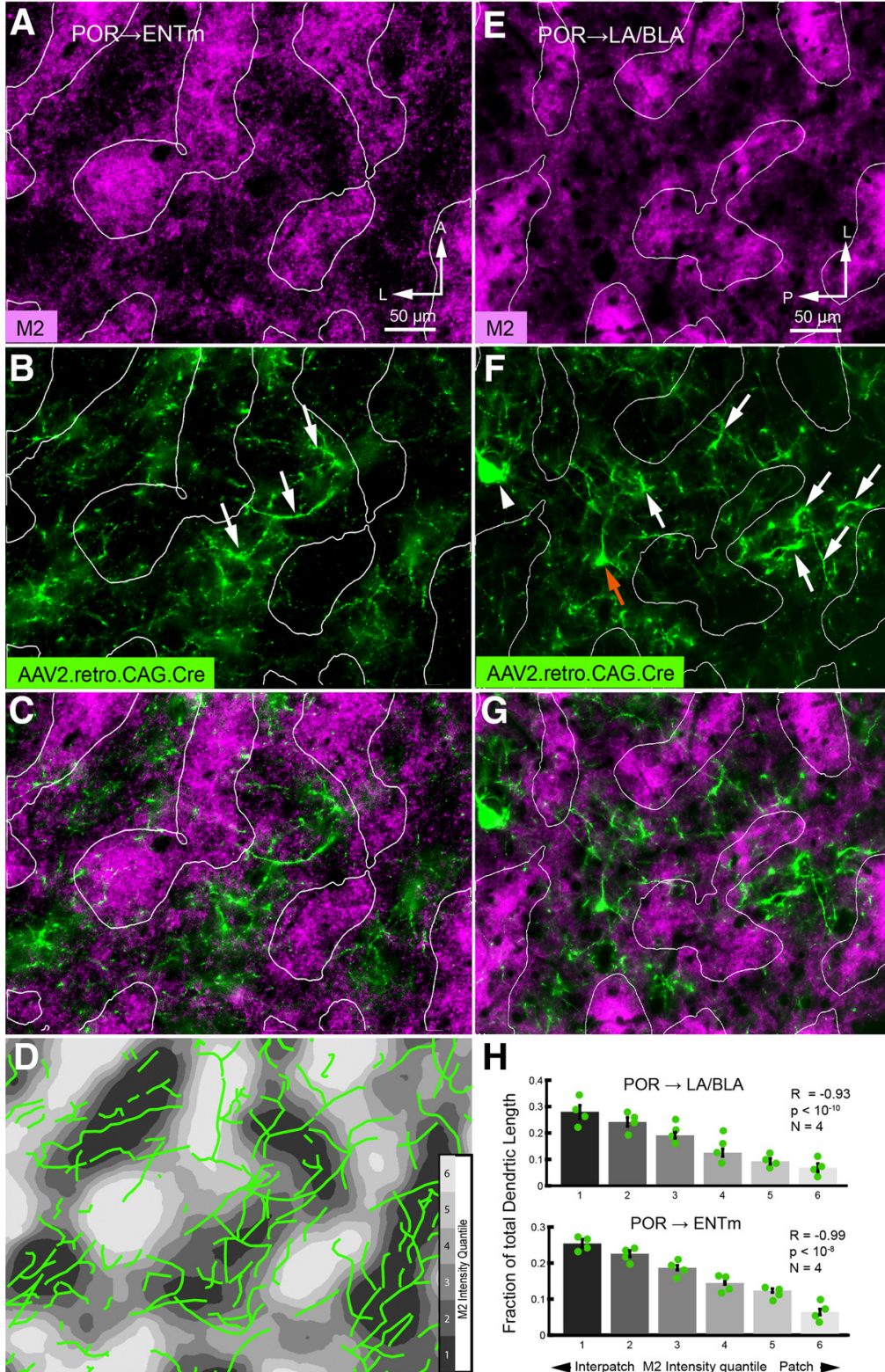

\section{H}
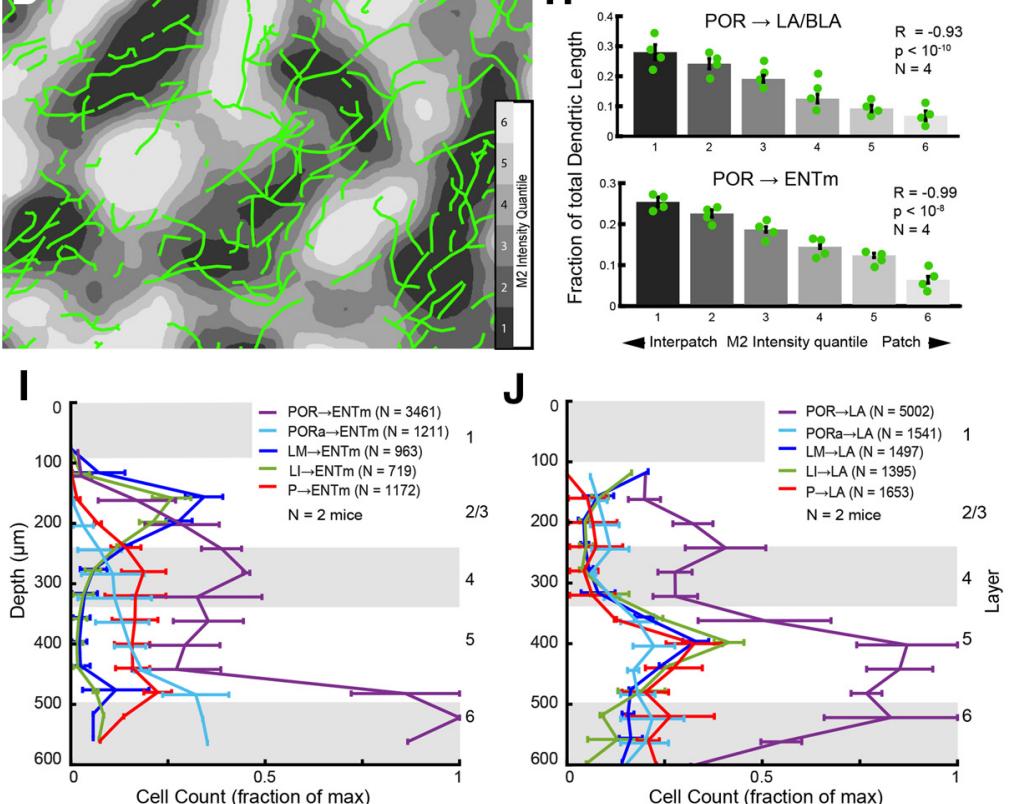

Figure 8. Modular distribution of retrogradely labeled apical dendrites of POR $\rightarrow$ ENTm and POR $\rightarrow$ amygdala projecting neurons in L1 of POR. A-C, Tangential section through flatmounted POR showing patchy expression of M2 immunostaining in L1 $(\boldsymbol{A})$ and nonuniform branching pattern of spine-covered apical dendrites (false-colored green, denoted by white arrows) in $\mathrm{L} 1$ of POR $\rightarrow$ ENTm projecting neurons labeled by retrograde tracing with $\mathrm{rAAV} 2-$ Retro/CAG-Cre in Ai9 mice $(\boldsymbol{B})$; overlay of $\boldsymbol{A}$ and $\boldsymbol{B}$ shows that dendritic branches are preferentially located in $\mathrm{ML}^{-}$interpatches (C). $\boldsymbol{D}$, Tracings of retrogradely labeled dendrites (green) in L1 of POR overlaid onto contour plot of M2 expression. Shades of gray represent six quantiles of fluorescence intensity. $\boldsymbol{E}, \boldsymbol{F}$, Tangential section through flatmounted POR showing patchy expression of $M 2$ immunostaining in $\mathrm{L} 1(\boldsymbol{E})$ and nonuniform branching pattern of apical dendrites (false-colored green) of POR $\rightarrow$ LA/BLA projecting neurons labeled by retrograde tracing with rAAV2-Retro/CAG-Cre $(\boldsymbol{F})$. White arrows indicate dendrites branching in the plane of the pial surface. Red arrow points to a thick vertically ascending dendritic trunk at were anisotropic and showed aspect ratios similar to the overall shape of the area (Garrett et al., 2014) in which they reside. Notably, we found a reduced (relative to V1) magnification factor per patch in LM $(12.5 \%$ smaller), POR (37.5\% smaller), and LI (50\% smaller), indicating that a module in each of these areas represents a larger portion of visual space than in V1. The results show that the magnification factor decreases as the receptive field size increases from $\mathrm{V} 1 \rightarrow \mathrm{LM}$ $\rightarrow$ LI $\rightarrow$ POR (D'Souza et al., 2020; Siegle et al., 2021), indicating that at the apex the product (i.e., point image) is represented by the smallest number of modules and remains constant. The findings further indicate that inputs from diverse retinotopic locations converge and that individual patches are processing units that integrate a broader set of stimulus attributes inherited from intracortical and subcortical sources (Beltramo and Scanziani, 2019; Harris et al., 2019; D’Souza et al., 2020).

POR contains separate module-specific circuits with dLGN, LP, amygdala, and ENTm

Our anterograde tracings with BDA have shown that only the ventral stream areas LM, LI, P, and POR provide input to the amygdala. No such connections were found from $\mathrm{V} 1$ and the dorsal stream areas AL, RL, A, AM, and PM. Conceptually similar results were reported in rat (McDonald and Mascagni, 1996). Together, the results suggest that only higher visual areas involved in encoding of objects and the context in which they appear have direct connections to the amygdala, while areas that play a role in visually guided actions (Jin and Glickfeld, 2020) do not. From this simplified perspective, it is easy to overlook that POR is highly interconnected with dorsal and ventral stream areas (Wang et al., 2012; Gămănuţ et al., 2018). Through these connections, POR combines task-specific information across streams,

the branchpoint of terminal tuft at the $L 1 / 2$ border. Because the section is not perfectly parallel to $L 1$ and cuts into $L 2$ it shows a retrogradely labeled cell body at the L1/2 border (arrowhead). $\mathbf{G}$, Overlay of $\boldsymbol{E}$ and $\boldsymbol{F}$ shows that dendritic branches (green) and cell bodies at the $L 1 / 2$ border are preferentially aligned with $M 2^{-}$ interpatches. $\boldsymbol{H}$, Length of dendritic branches of POR $\rightarrow L A / B L A$ and $\mathrm{POR} \rightarrow$ ENTm projecting neurons in $\mathrm{M2}^{+}$patches and $\mathrm{M}^{-}$ interpatches of $L 1$ of POR. The fraction (mean \pm SEM) of total dendritic lengths for both types of neurons shows a strong (POR $\rightarrow$ LA/BLA: $R=-0.93, p<10^{-10}, N=4$ mice; POR $\rightarrow$ ENTm: $R=-0.99, p<10^{-8}, N=4$ mice, Pearson correlation) preference for $\mathrm{ML}^{-}$interpatches. $I, J$, Laminar distribution (mean \pm SEM) of retrogradely labeled cells in POR, PORa, LM, LI, and P projecting to ENTm (I) and LA/BLA (I), respectively. Same axes in $I$ and $J$. 

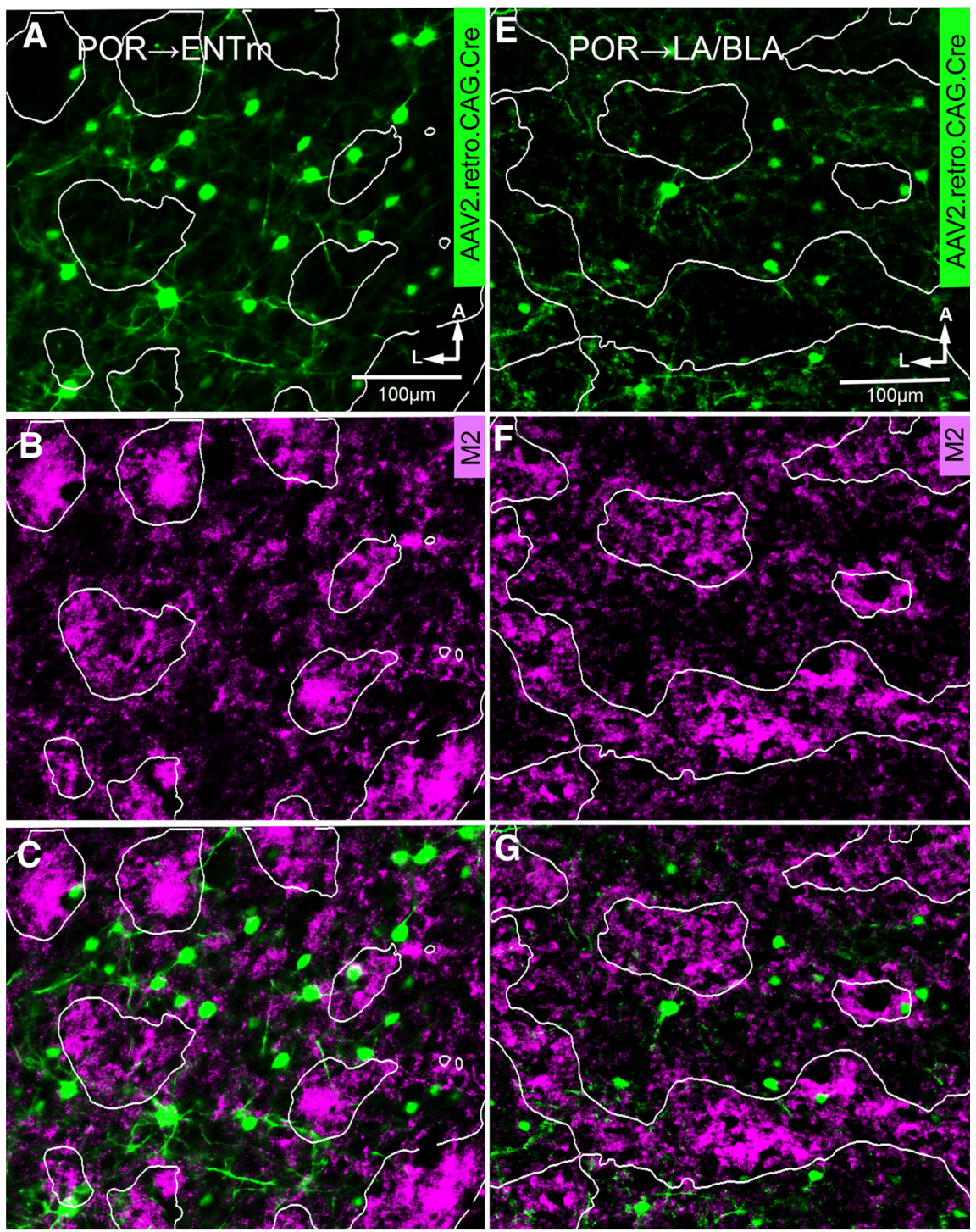

D

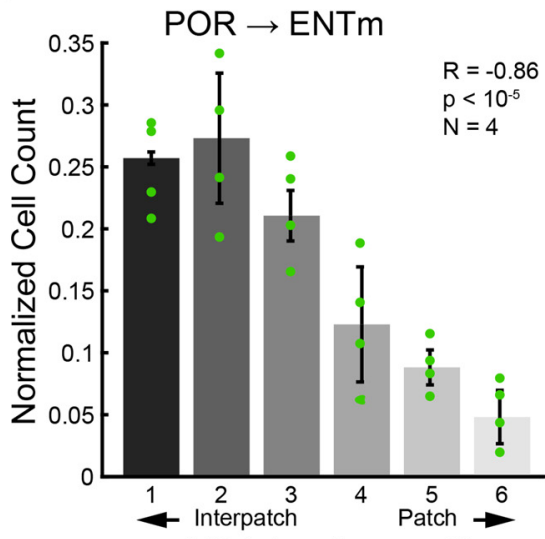

M2 Intensity quantile
H

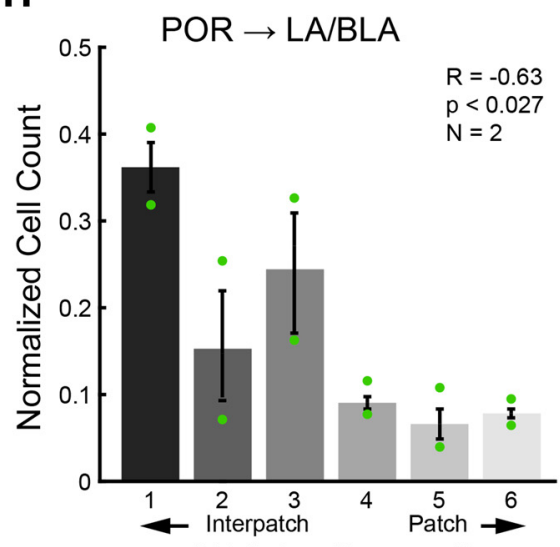

M2 Intensity quantile

Figure 9. Modular distribution of retrogradely labeled cell bodies of POR $\rightarrow L A / B L A$ and POR $\rightarrow$ ENTm projecting neurons in L2/3 of POR. $\boldsymbol{A}, \boldsymbol{B}$, Tangential section through flatmounted POR showing nonuniform distribution of somata (false-colored green) of POR $\rightarrow$ ENTm projecting L2/3 neurons labeled by retrograde tracing with rAAV2-Retro/CAG-Cre in Ai 9 mice (A) overlaid with patchy expression of M2 immunostaining in $\mathrm{L} 1(\boldsymbol{B})$. $\boldsymbol{C}$, Overlay of $\boldsymbol{A}$ and $\boldsymbol{B}$ shows that cell bodies and basal dendrites are preferentially aligned with $\mathrm{M2}^{-}$interpatches. $\boldsymbol{D}$, Normalized cell counts (mean \pm SEM) of POR $\rightarrow$ ENTm in M2 quantiles (shades of gray) of POR. The fraction of total cell bodies shows a strong preference for M2 ${ }^{-}$interpatches. $E-G$, Tangential section through flatmounted POR showing nonuniform distribution of somata (false-colored green) of POR $\rightarrow$ LA/BLA projecting L2/3 neurons labeled by retrograde tracing with rAAV2-Retro/CAG-Cre in Ai 9 mice $(\boldsymbol{E})$ overlaid with patchy expression of M2 immunostaining in L1 $(\boldsymbol{F})$. $\boldsymbol{G}$, Overlay of $\boldsymbol{E}$ and $\boldsymbol{F}$ shows that cell bodies are preferentially aligned with $\mathrm{ML}^{-}$interpatches. $\boldsymbol{H}$, Normalized cell counts (mean $\pm S E M$ ) of POR $\rightarrow$ LA/BLA in M2 quantiles of POR. The fraction of total cell bodies shows a preference for M2 ${ }^{-}$interpatches. 

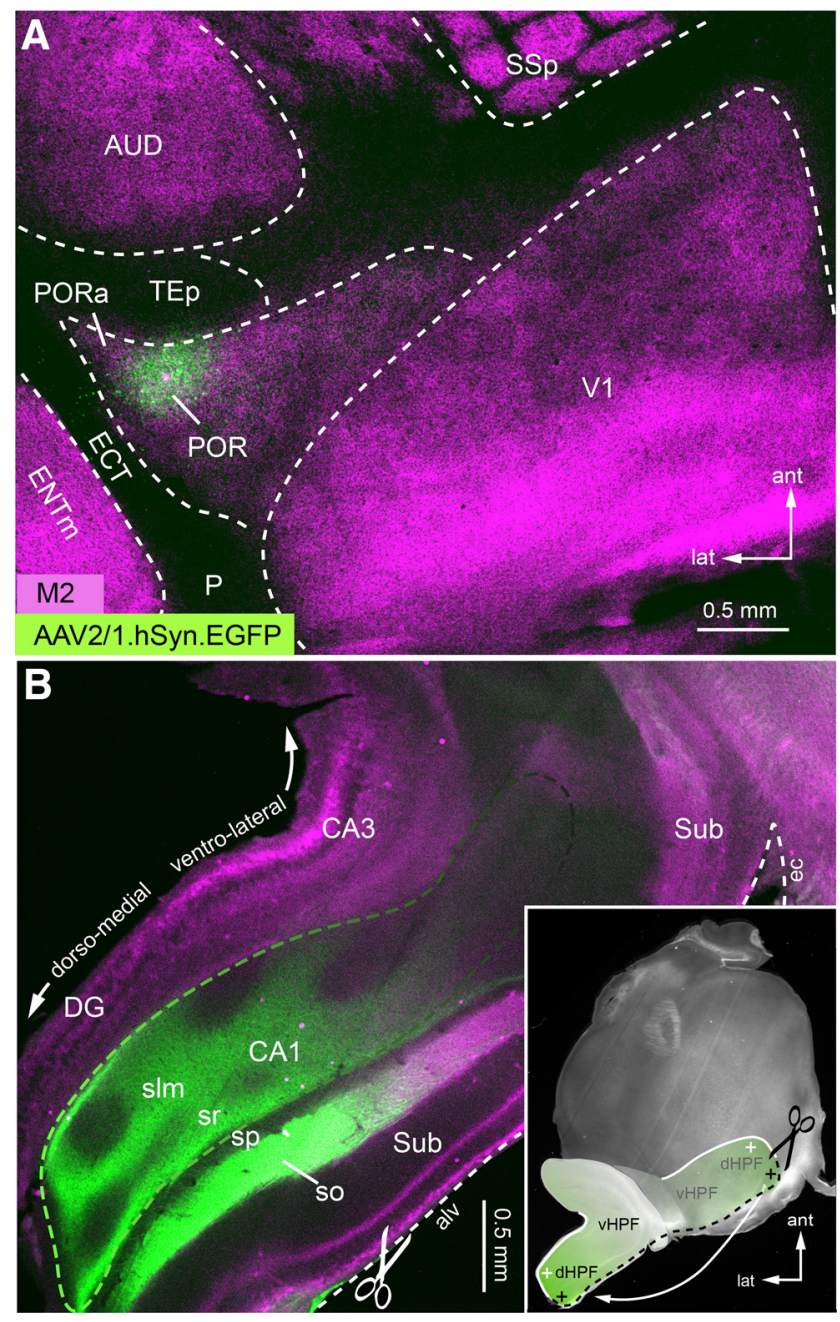

Figure 10. Anterogradely labeled POR inputs to CA1 of hippocampus. A, Tangential section through L4 of flatmounted cortex stained with an antibody against M2 (magenta). Green spot indicates injection site of AAV2/1.hSyn.EGFP in POR. $\boldsymbol{B}$, Tangential section through flatmounted HPF. For flatmounting, the hippocampus was separated from the external capsule (ec) along the alveus (alv) and flipped backward (inset) so that the ventral side faced the pial surface and the dorsal pole (cross hairs) came to lie at the posterior edge of the section through the flatmounted left cortical hemisphere (inset). POR $\rightarrow$ CA1 axons (green) are most densely labeled at the dorsal pole and gradually fade away toward the ventral pole. DG, Dentrate gyrus; slm, stratum lacunosum moleculare; so, stratum oriens; sp, stratum pyramidale; sr, stratum radiale; Sub, subiculum; dHPF, dorsal HPF; vHPF, ventral HPF.

associates objects with locations (i.e., context; Furtak et al., 2012), and, through interactions with the amygdala, assigns affective credits to neurons that guide behavior (Bucci and Burwell, 2004; Burgess et al., 2016). Notably, only POR sends significant input to the BLA nucleus, which projects onward to the CeA (Pitkänen et al., 1995) and is the major driver of behavioral responses from the amygdala (Tye et al., 2011). Thus, it appears that POR has more direct control over visually related affective behaviors than areas LM, LI, and P, whose projections are weak and largely confined to LA.

The patchy pattern of M2 expression we have found in L1 of areas LM, LI, POR, and PORa reveals that the modular organization of V1 (Ji et al., 2015) is preserved across visual areas of the ventral stream (Wang et al., 2012). Modularity, in the sense used here, refers to the columnar organization of stimulus-selective responses and the spatial clustering of projection neurons within an area (da Costa and Martin, 2010). That such constraints emerge from the segmentation of the plexiform sheet of L1 has only been shown in V1, where a patchy pattern of M2 expression label sites of thalamocortical and intracortical synaptic input to

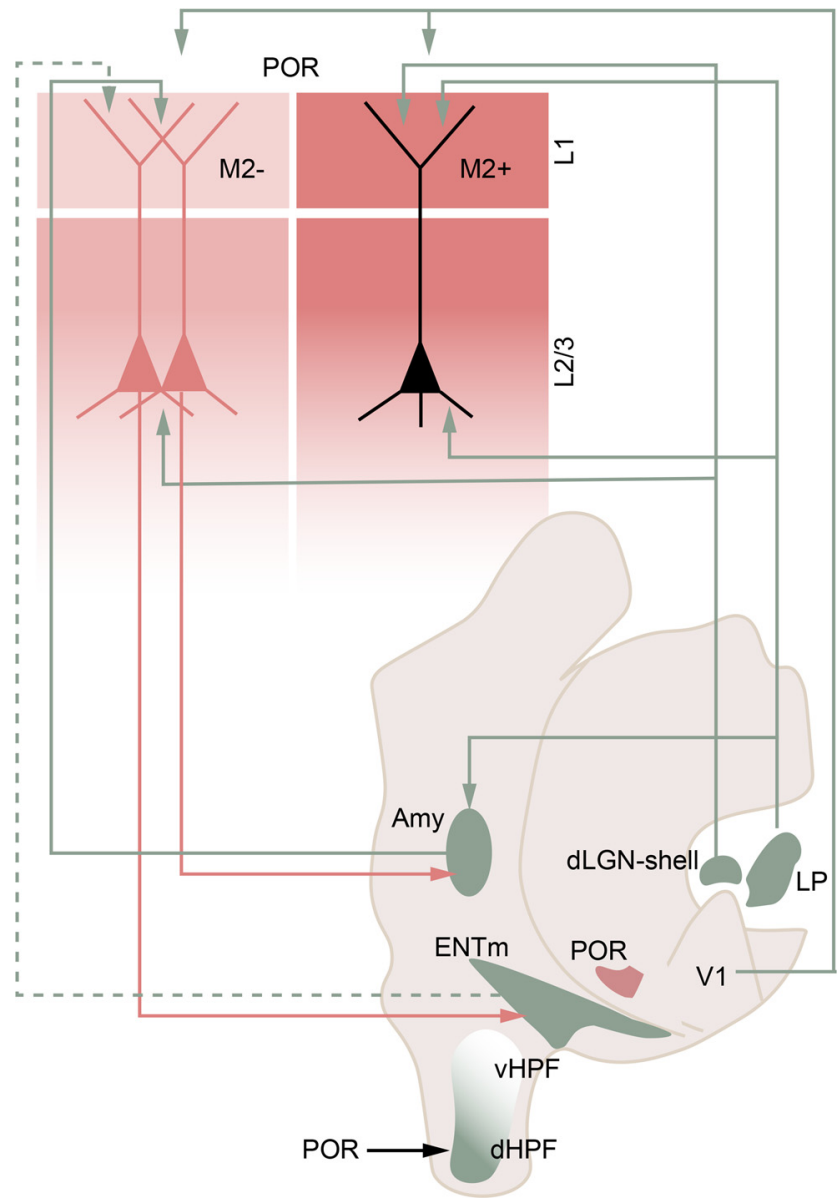

Figure 11. Circuit diagram of the modular thalamo-amygdalo-entorhinal network with POR. Modules in POR are depicted as $\mathrm{M2}^{+}$patches (dark red) and $\mathrm{ML}^{-}$interpatches (light red) in $\mathrm{L} 1$, which indicate clusters or putative columns across the layers below. The M2-defined modularity is supported by patch- and interpatch-specific inputs and outputs. Inputs from the dLGN shell and the lateral posterior nucleus (LP or pulvinar) go to $\mathrm{M}^{+}$patches. Inputs from V1 to POR are equally dense in $\mathrm{M2}^{+}$patches and $\mathrm{ML}^{-}$interpatches. Inputs from the amygdala (Amy) and, hypothetically, the ENTm (dotted line) terminate in $\mathrm{ML}^{-}$interpatches. $\mathrm{ML}^{-}$interpatches also contain the dendrites and cell bodies (red) of POR neurons projecting to Amy and ENTm. The projection targets of patch neurons (black) are unknown. POR projections to the HPF are biased toward the dorsal pole (dHPF) and are undetectable in the ventral part (VHPF). It is unknown whether POR $\rightarrow$ HPF neurons are preferentially associated $\mathrm{ML}^{+}$patches or $\mathrm{ML}^{-}$interpatches or reside in both modules.

apical dendrites of pyramidal neurons (Ji et al., 2015; D'Souza et al., 2019). We have found that similar patchy patterns in L1 organize the modularity of amygdalocortical and thalamocortical inputs to POR, PORa, LM, and LI. The results show that of all layers the LA innervates L1 most strongly and preferentially targets $\mathrm{M}^{-}$interpatches. Thalamic inputs from dLGN and LP to POR, PORa, LM, and LI not only differ from amygdalocortical afferents in their laminar distribution, but also in their preferential targeting of $\mathrm{M}^{+}$patches in $\mathrm{L} 1$. The distinctive connectivity patterns suggest that amygdalocortical and thalamocortical inputs to L1 target apical dendrites of separate populations of POR and PORa neurons, whose cell bodies are located in the layers below. Spatial clustering of pyramidal cell dendritic tufts in $\mathrm{L} 1$ are known features of rat V1 and retrosplenial cortex (Ichinohe and Rockland, 2002; Ichinohe et al., 2003). Here, we demonstrate directly that the apical dendrites of POR $\rightarrow$ amygdala projecting neurons preferentially terminate in $\mathrm{M}^{-}$interpatches. Thus, it is tempting to speculate that pyramidal cells in POR with dendrites in $\mathrm{M}^{-}$interpatches may be modulated by affective information from the amygdala (Bucci et al., 2000; 
Burgess et al., 2016), which is driven directly by visual input from LP (Wei et al., 2015). In contrast, responses of POR neurons with dendrites in $\mathrm{M}^{+}$patches may be influenced preferentially by direct locomotion-modulated inputs from the dLGN shell and LP (Marshel et al., 2012; Roth et al., 2016; Beltramo and Scanziani, 2019; Bennett et al., 2019) rather than by inputs ascending through the hierarchical cortical network originating in V1 (Beltramo and Scanziani, 2019; D'Souza et al., 2020).

The overlap between dendrites of POR output neurons with inputs from the amygdala suggests that the amygdala and POR are interconnected through a reciprocal loop, as has been shown directly by Burgess et al. (2016). It seems likely that these reward-modulated responses are mediated by synaptic contacts in L1. Tagging sensory stimuli with affective significance imported from the amygdala is not the only influence on the multimodal response profile of POR neurons, which are also sensitive to the spatial context in which objects appear (Furtak et al., 2012). This information may derive from dorsal stream inputs (Wang et al., 2012; Gămănuţ et al., 2018) and is sent to multiple downstream targets (Agster et al., 2016), including the ENTm, with which we have found POR to be reciprocally connected. Similar to POR $\rightarrow$ amygdala projecting cells, the apical dendrites of POR $\rightarrow$ ENTm neurons terminate preferentially in $\mathrm{M}^{-}$interpatches. These dendrites may belong to $\mathrm{POR} \rightarrow \mathrm{LA} / \mathrm{BLA}$ and $\mathrm{POR} \rightarrow$ ENTm cells or belong to the same neurons with branching projections to both the amygdala and ENTm. Regardless, the results suggests that some POR neurons with dendrites in $\mathrm{M} 2^{-}$interpatches send affect-modulated outputs to ENTm, where they may enhance the emotional salience of landmark information and unexpected objects in the external world (Knierim et al., 2014), and guide defensive responses to visual threats (Bucci and Burwell, 2004; Wei et al., 2015). In addition to this indirect pathway, POR also provides direct input to the dorsal HPF, suggesting that it serves visuospatial processing for environmental exploration (Fanselow and Dong, 2010). Although the modular origin of POR $\rightarrow$ HPF neurons remains unknown, the sparse innervation of intermediate and ventral HPF suggests that the input may carry little affective information (Fanselow and Dong, 2010).

In contrast to amygdala $\rightarrow$ POR projections, we found that $\mathrm{dLGN} \rightarrow \mathrm{POR}$ and $\mathrm{LP} \rightarrow$ POR inputs overlap with $\mathrm{M}^{+}$patches of L1. Retrograde labeling from POR confirmed that dLGN inputs originate from shell neurons, which are known to receive afferents from the retina and the SC (Dhande and Huberman, 2014; Morin and Studholme, 2014; Bickford et al., 2015; Martersteck et al., 2017) and, like the $\mathrm{SC} \rightarrow \mathrm{LP} \rightarrow$ POR pathway, convey visual motion information to its target (Cruz-Martín et al., 2014; Beltramo and Scanziani, 2019; Bennett et al., 2019). Inputs from matrix-type thalamic neurons to dendrites in L1 have been shown to elicit spiking in pyramidal cells of rat barrel cortex (Cruikshank et al., 2010) and may possess similar synaptic strength in POR. Both pathways carry locomotion signals, in which running speed is either synchronized or desynchronized with optic flow motion (Roth et al., 2016). Synchronous convergent thalamocortical inputs, heavily biased from $\mathrm{LP}$, to $\mathrm{M}^{+}$patches in POR may mostly signal moving objects at speeds different from the motion of the animals. These direct thalamic inputs to apical dendrites in $\mathrm{M}^{+}$patches of POR may increase the sensitivity of POR neurons (Takahashi et al., 2016) to nonthreatening moving stimuli independent of the hierarchical cortical circuitry (Beltramo and Scanziani, 2019). Future studies are necessary to determine to which circuit $\mathrm{M}^{+}$patch-aligned POR cells belong (Fig. 11).

\section{References}

Agster KL, Tomás Pereira I, Saddoris MP, Burwell RD (2016) Subcortical connections of the perirhinal, postrhinal, and entorhinal cortices of the rat. II. Efferents. Hippocampus 26:1213-1230.

Arlotta P, Molyneaux BJ, Chen J, Inoue J, Kominami R, Macklis JD (2005) Neuronal subtype-specific genes that control corticospinal motor neuron development in vivo. Neuron 45:207-221.

Beaudin SA, Singh T, Agster KL, Burwell RD (2013) Borders and comparative cytoarchitecture of the perirhinal and postrhinal cortices in an F1 hybrid mouse. Cereb Cortex 23:460-476.

Beltramo R, Scanziani M (2019) A collicular visual cortex: neocortical space for an ancient midbrain visual structure. Science 363:64-69.

Bennett C, Gale SD, Garrett ME, Newton ML, Callaway EM, Murphy GJ, Olsen SR (2019) Higher-order thalamic circuits channel parallel streams of visual information in mice. Neuron 102:477-492.

Bickford ME, Zhou N, Krahe TE, Govindaiah G, Guido W (2015) Retinal and tectal "driver-like" inputs converge in the shell of the mouse dorsal lateral geniculate nucleus. J Neurosci 35:10523-10534.

Bucci DJ, Burwell RD (2004) Deficits in attentional orienting following damage to the perirhinal or postrhinal cortices. Behav Neurosci 118:1117-1122.

Bucci DJ, Phillips RG, Burwell RD (2000) Contributions of postrhinal and perirhinal cortex to contextual information processing. Behav Neurosci 114:882-894.

Burgess CR, Ramesh RN, Sugden AU, Levandowski KM, Minnig MA, Fenselau H, Lowell BB, Andermann ML (2016) Hunger-dependent enhancement of food cue responses in mouse postrhinal cortex and lateral amygdala. Neuron 91:1154-1169.

Burwell RD (2001) Borders and cytoarchitecture of the perirhinal and postrhinal cortices in the rat. J Comp Neurol 437:17-41.

Burwell RD, Witter MP, Amaral DG (1995) Perirhinal and postrhinal cortices of the rat: a review of the neuroanatomical literature and comparison with findings from the monkey brain. Hippocampus 5:390-408.

Buzsáki G, Moser EI (2013) Memory, navigation and theta rhythm in the hippocampal-entorhinal system. Nat Neurosci 16:130-138.

Cruikshank SJ, Urabe H, Nurmikko AV, Connors BW (2010) Pathway-specific feedforward circuits between thalamus and neocortex revealed by selective optical stimulation of axons. Neuron 65:230-245.

Cruz-Martín A, El-Danaf RN, Osakada F, Sriram B, Dhande OS, Nguyen PL, Callaway EM, Ghosh A, Huberman AD (2014) A dedicated circuit links direction-selective retinal ganglion cells to the primary visual cortex. Nature 507:358-361.

D’Souza RD, Bista P, Meier AM, Ji W, Burkhalter A (2019) Spatial clustering of inhibition in mouse primary visual cortex. Neuron 104:588-600.

D'Souza RD, Wang Q, Ji W, Meier AM, Kennedy H, Knoblauch K, Burkhalter A (2020) Canonical and noncanonical features of the mouse visual cortical hierarchy. bioRxiv 016303. doi: 10.1101/2020.03.30. 016303.

da Costa NMM, Martin KAC (2010) Whose cortical column would that be? Front Neuroanat 4:16

Deshmukh SS, Johnson JL, Knierim JJ (2012) Perirhinal cortex represents nonspatial, but not spatial, information in rats foraging in the presence of objects: comparison with lateral entorhinal cortex. Hippocampus 22:2045-2058.

Dhande OS, Huberman AD (2014) Retinal ganglion cell maps in the brain: implications for visual processing. Curr Opin Neurobiol 24:133-142.

Dong HW (2008) The Allen reference atlas: a digital color brain atlas of the C57Bl/6J male mouse. Hoboken, NJ: Wiley.

Epstein R, Harris A, Stanley D, Kanwisher N (1999) The parahippocampal place area. Neuron 23:115-125.

Fanselow MS, Dong H-W (2010) Are the dorsal and ventral hippocampus functionally distinct structures? Neuron 65:7-19.

Floyd NS, Price JL, Ferry AT, Keay KA, Bandler R (2000) Orbitomedial prefrontal cortical projections to distinct longitudinal columns of the periaqueductal gray in the rat. J Comp Neurol 422:556-578.

Franklin BJ, Paxinos G (2008) The mouse brain in stereotaxic coordinates, Ed 3. Amsterdam: Academic, Elsevier.

Furtak SC, Ahmed OJ, Burwell RD (2012) Single neuron activity and theta modulation in postrhinal cortex during visual object discrimination. Neuron 76:976-988.

Gămănuţ R, Kennedy $\mathrm{H}$, Toroczkai Z, Ercsey-Ravasz M, Essen DCV, Knoblauch K, Burkhalter A (2018) The Mouse cortical connectome, characterized by an ultra-dense cortical graph, maintains specificity by distinct connectivity profiles. Neuron 97:698-715. 
Garrett ME, Nauhaus I, Marshel JH, Callaway EM (2014) Topography and areal organization of mouse visual cortex. J Neurosci 34:12587-12600.

Gofman X, Tocker G, Weiss S, Boccara CN, Lu L, Moser M-B, Moser EI, Morris G, Derdikman D (2019) Dissociation between postrhinal cortex and downstream parahippocampal regions in the representation of egocentric boundaries. Curr Biol 29:2751-2757.

Harris JA, Mihalas S, Hirokawa KE, Whitesell JD, Choi H, Bernard A, Bohn P, Caldejon S, Casal L, Cho A, Feiner A, Feng D, Gaudreault N, Gerfen CR, Graddis N, Groblewski PA, Henry AM, Ho A, Howard R, Knox JE, Kuan L, et al. (2019) Hierarchical organization of cortical and thalamic connectivity. Nature 575:195-202.

Hintiryan H, Bowman I, Johnson DL, Korobkova L, Zhu M, Khanjani N, Gou L, Gao L, Yamashita S, Bienkowski MS, Garcia L, Foster NN, Benavidez NL, Song MY, Lo D, Cotter K, Becerra M, Aquino S, Cao C, Cabeen R, et al. (2019) Connectivity characterization of the mouse basolateral amygdalar complex. bioRxiv 807743. doi: 10.1101/807743.

Hunnicutt BJ, Jongbloets BC, Birdsong WT, Gertz KJ, Zhong H, Mao T (2016) A comprehensive excitatory input map of the striatum reveals novel functional organization. Elife 5:5e19103.

Ichinohe N, Rockland KS (2002) Parvalbumin positive dendrites co-localize with apical dendritic bundles in rat retrosplenial cortex. Neuroreport 13:757-761.

Ichinohe N, Fujiyama F, Kaneko T, Rockland KS (2003) Honeycomb-like mosaic at the border of layers 1 and 2 in the cerebral cortex. J Neurosci 23:1372-1382.

Janak PH, Tye KM (2015) From circuits to behaviour in the amygdala. Nature 517:284-292.

Ji W, Gămănuţ R, Bista P, D’Souza RD, Wang Q, Burkhalter A (2015) Modularity in the organization of mouse primary visual cortex. Neuron 87:632-643

Jin M, Glickfeld LL (2020) Mouse higher visual areas provide both distributed and specialized contributions to visually guided behaviors. Curr Biol 30:4682-4692.e7.

Jones EG (2007) The thalamus, Ed 2. Cambridge, UK: Cambridge UP.

Juavinett AL, Kim EJ, Collins HC, Callaway EM (2020) A systematic topographical relationship between mouse lateral posterior thalamic neurons and their visual cortical projection targets. J Comp Neurol 528:99-111.

Kaas JH (2012) Evolution of columns, modules, domains in the neocortex of primates. Proc Natl Acad Sci U S A 109:10655-10660.

Karimi A, Odenthal J, Drawitsch F, Boergens KM, Helmstaedter M (2020) Cell-type specific innervation of cortical pyramidal cells at their apical dendrites. Elife 9:e46876.

Knierim JJ, Neunuebel JP, Deshmukh SS (2014) Functional correlates of the lateral and medial entorhinal cortex: objects, path integration and local-global reference frames. Philos Trans R Soc Lond B Biol Sci 369:20130369.

LaChance PA, Todd TP, Taube JS (2019) A sense of space in postrhinal cortex. Science 365:eaax4192.

Lavenex P, Suzuki WA, Amaral DG (2004) Perirhinal and parahippocampal cortices of the macaque monkey: intrinsic projections and interconnections. J Comp Neurol 472:371-394.

LeDoux J (2012) Rethinking the emotional brain. Neuron 73:653-676.

Marshel JH, Kaye AP, Nauhaus I, Callaway EM (2012) Anterio-posterior direction opponency in the superficial mouse lateral geniculate nucleus. Neuron 76:713-720.

Martersteck EM, Hirokawa KE, Evarts M, Bernard A, Duan X, Li Y, Ng L, Oh SW, Ouellette B, Royall JJ, Stoecklin M, Wang Q, Zeng H, Sanes JR, Harris JA (2017) Diverse central projection patterns of retinal ganglion cells. Cell Rep 18:2058-2072.

McDonald AJ, Mascagni F (1996) Cortico-cortical and cortico-amygdaloid projections of the rat occipital cortex: a Phaseolus vulgaris leucoagglutinin study. Neuroscience 71:37-54.

Morin LP, Studholme KM (2014) Retinofugal projections in the mouse. J Comp Neurol 522:3733-3753.

Nilssen ES, Doan TP, Nigro MJ, Ohara S, Witter MP (2019) Neurons and networks in the entorhinal cortex: a reappraisal of the lateral and medial entorhinal subdivisions mediating parallel cortical pathways. Hippocampus 29:1238-2254.
Nishio N, Tsukano H, Hishida R, Abe M, Nakai J, Kawamura M, Aiba A, Sakimura K, Shibuki K (2018) Higher visual responses in the temporal cortex of mice. Sci Rep 8:11136.

Oh SW, Harris JA, Ng L, Winslow B, Cain N, Mihalas S, Wang Q, Lau C, Kuan L, Henry AM, Mortrud MT, Ouellette B, Nguyen TN, Sorensen SA, Slaughterbeck CR, Wakeman W, Li Y, Feng D, Ho A, Nicholas E, et al (2014) A mesoscale connectome of the mouse brain. Nature 508:207-214.

Pessoa L, Adolphs R (2011) Emotion and the brain: multiple roads are better than one. Nat Rev Neurosci 12:425-425.

Phelps EA, LeDoux JE (2005) Contributions of the amygdala to emotion processing: from animal models to human behavior. Neuron 48:175-187.

Pikkarainen M, Pitkänen A (2001) Projections from the lateral, basal and accessory basal nuclei of the amygdala to the perirhinal and postrhinal cortices in rat. Cereb Cortex 11:1064-1082.

Pitkänen A, Stefanacci L, Farb CR, Go G-G, Ledoux JE, Amaral DG (1995) Intrinsic connections of the rat amygdaloid complex: projections originating in the lateral nucleus. J Comp Neurol 356:288-310.

Price JL (2005) Free will versus survival: brain systems that underlie intrinsic constraints on behavior. J Comp Neurol 493:132-139.

Ramesh RN, Burgess CR, Sugden AU, Gyetvan M, Andermann ML (2018) Intermingled ensembles in visual association cortex encode stimulus identity or predicted outcome. Neuron 100:900-915.e9.

Roth MM, Dahmen JC, Muir DR, Imhof F, Martini FJ, Hofer SB (2016) Thalamic nuclei convey diverse contextual information to layer 1 of visual cortex. Nat Neurosci 19:299-307.

Saleem KS, Price JL, Hashikawa T (2007) Cytoarchitectonic and chemoarchitectonic subdivisions of the perirhinal and parahippocampal cortices in macaque monkeys. J Comp Neurol 500:973-1006.

Sereno MI, Allman J (1991) Cortical visual areas in mammals. In: The basis of visual function (Leventhal AG, ed), pp 160-172. London: Macmillan.

Sherman SM (2016) Thalamus plays a central role in ongoing cortical functioning. Nat Neurosci 19:533-541.

Siegle JH, Jia X, Durand S, Gale S, Bennett C, Graddis N, Heller G, Ramirez K, Choi H, Milhalas S, Luviano JA, Groblewski PA, Ahmed R, Arkhipov A, Bernard A, Billeh YN, Brown D, Buice M, Cain N, Caldejon S, et al (2021) A survey of spiking activity reveals a functional hierarchy of mouse corticothalamic visual areas. Nature 592:86-92.

Sincich LC, Horton JC (2005) Input to V2 thin stripes arise from V1 cytochrome oxidase patches. J Neurosci 25:10087-10093.

Swanson LW, Hahn JD (2020) A qualitative solution with quantitative potential for the mouse hippocampal cortex flatmap problem. Proc Natl Acad Sci U S A 117:3220-3231.

Takahashi N, Oertner TG, Hegemann P, Larkum ME (2016) Active cortical dendrites modulate perception. Science 354:1587-1590.

Tye KM, Prakash R, Kim S-Y, Fenno LE, Grosenick L, Zarabi H, Thompson KR, Gradinaru V, Ramakrishnan C, Deisseroth K (2011) Amygdala circuitry mediating reversible and bidirectional control of anxiety. Nature 471:358-362.

Ungerleider LG, Galkin TW, Desimone R, Gattass R (2008) Cortical connections of area V4 in the macaque. Cereb Cortex 18:477-499.

Vaughan DW, Peters A (1973) A three dimensional study of layer I of the rat parietal cortex. J Comp Neurol 149:355-370.

Wang Q, Burkhalter A (2007) Area map of mouse visual cortex. J Comp Neurol 502:339-357.

Wang Q, Gao E, Burkhalter A (2011) Gateways of ventral and dorsal streams in mouse visual cortex. J Neurosci 31:1905-1918.

Wang Q, Sporns O, Burkhalter A (2012) Network analysis of corticocortical connections reveals ventral and dorsal processing streams in mouse visual cortex. J Neurosci 32:4386-4399.

Wei P, Liu N, Zhang Z, Liu X, Tang Y, He X, Wu B, Zhou Z, Liu Y, Li J, Zhang Y, Zhou X, Xu L, Chen L, Bi G, Hu X, Xu F, Wang L (2015) Processing of visually evoked innate fear by a non-canonical thalamic pathway. Nat Commun 6:6756.

Zhou N, Masterson SP, Damron JK, Guido W, Bickford ME (2017) The mouse pulvinar nucleus links the lateral extrastriate cortex, striatum, amygdala. J Neurosci 38:347-362.

Zingg B, Hintiryan H, Gou L, Song MY, Bay M, Bienkowski MS, Foster NN, Yamashita S, Bowman I, Toga AW, Dong H-W (2014) Neural networks of the mouse neocortex. Cell 156:1096-1111. 